\title{
Retrospective review of task-shifting community-based programs supporting ARV treatment and retention in Uganda
}

\author{
Sam Kalibala \\ Population Council \\ Jerry Okal \\ Population Council \\ Brady Zieman \\ Population Council \\ Nrupa Jani \\ Population Council \\ Lung Vu \\ Population Council
}

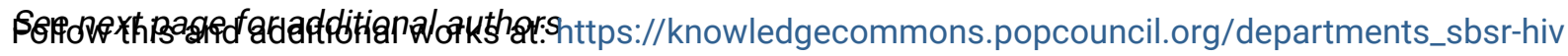

Part of the Demography, Population, and Ecology Commons, Family, Life Course, and Society

Commons, and the International Public Health Commons

How does access to this work benefit you? Let us know!

\section{Recommended Citation}

Kalibala, Sam, Jerry Okal, Brady Zieman, Nrupa Jani, Lung Vu, Josephine Birungi, Stephen Okoboi, Jonathan Wangisi, Christine Nabiryo, Joanne Lyavala Okullu, Michael Etukoit, Lydia Buzaalirwa, Augustine Lubanga, Paul Kiyingi, Jonathan Ikapule, Syson Nakyeyune, Stephen Nakibinge, Penninah Lutung, Samuel Waliggo, Joseph Nsamba, Yashien Wamanga, Joseph Kamya, Robina Sentongo, and Robert Yiga. 2016. "Retrospective review of task-shifting community-based programs supporting ARV treatment and retention in Uganda," HIVCore Final Report. Washington, DC: USAID I Project Search: HIVCore. 


\section{Authors}

Sam Kalibala, Jerry Okal, Brady Zieman, Nrupa Jani, Lung Vu, Josephine Birungi, Stephen Okoboi, Jonathan Wangisi, Christine Nabiryo, Joanne Lyavala Okullu, Michael Etukoit, Lydia Buzaalirwa, Augustine Lubanga, Paul Kiyingi, Jonathan Ikapule, Syson Nakyeyune, Stephen Nakibinge, Penninah Lutung, Samuel Waliggo, Joseph Nsamba, Yashien Wamanga, Joseph Kamya, Robina Sentongo, and Robert Yiga 
$\underline{\underline{\underline{\underline{\underline{E}}}} Q}$

PEPFÁR

O) USAID Project SEARCH

\section{HIVCore}

\section{RETROSPECTIVE REVIEW OF}

TASK-SHIFTING COMMUNITY-

BASED PROGRAMS SUPPORTING

ARV TREATMENT AND

RETENTION IN UGANDA

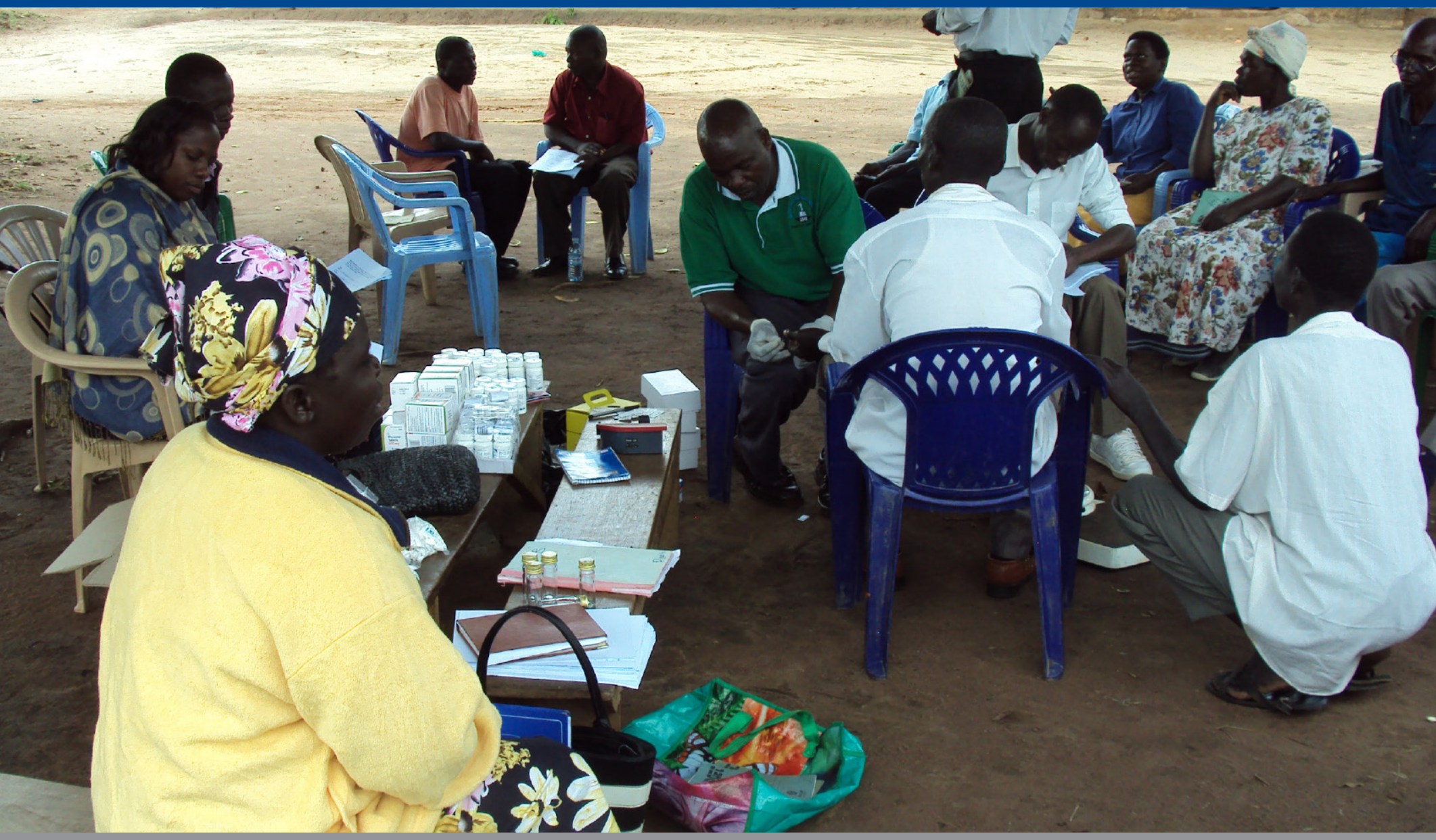

21 January 2016

This publication was produced for the United States Agency for International Development. It was prepared by S. Kalibala, S., J. Okal, B. Zieman, N. Jani, L.Vu, L.Ssali, J. Birungi, S. Okoboi, J.Wangisi, C. Nabiryo, J. Lyavala, M. Etukoit, L. Buzaalirwa, A. Lubanga, P. Kiyingi, J. Ikapule, S. Nakyeyune, S. Nakibinge, P. lutung, S. Waliggo, J. Nsamba, Y.Wamanga, J. Kamya, R. Sentongo, and R.Yiga. 



\section{RETROSPECTIVE REVIEW OF TASK-SHIFTING COMMUNITY-BASED PROGRAMS SUPPORTING ARVTREATMENT AND RETENTION IN UGANDA}

POPULATION COUNCIL: S. KALIBALA, J. OKAL, B. ZIEMAN, N. JANI, L.VU TASO: L. SSALI, J. BIRUNGI, S. OKOBOI, J.WANGISI, C. NABIRYO, J. LYAVALA, M. ETUKOIT

UGANDA CARES: L. BUZAALIRWA, A. LUBANGA, P. KIYINGI, J. IKAPULE, S. NAKYEYUNE, P.IUTUNG

KITOVU MOBILE: S.WALIGGO, J. NSAMBA, Y.WAMANGA, J. KAMYA, R. SENTONGO, R.YIGA

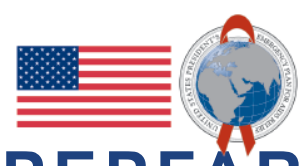

PEPFAR 


\section{ACKNOWLEDGMENTS}

The study team would like to highlight the key role played by the patients and service providers of the three AIDS service organizations (ASOs) — Kitovu Mobile, Uganda Cares, and TASO_that provided data for this study by participating in the surveys and interviews. In addition the work of the senior and mid-level managers of the three ASOs in the conceptualization of the study, organization and coordination of data collection, and participation in data interpretation meetings is highly appreciated. The study coordinator, Ms. Doreen Asaba, and the entire data collection and data entry team worked tirelessly to ensure the success of this study.

The reviewers of this report who included internal reviewers-Drs. Irit Sinai and Scott Geibel—and external peer reviewers-Drs. Avina Sarna and Francis Obare-made a great contribution to the quality of this report through their honest comments and constructive criticism. The USAID Task Order Management Team, Ms. Sarah Sandison and Dr. Glenn Post together with the USAID Technical Adviser on HIV Treatment Dr. Tom Minior, made significant contributions to this study by reviewing the concept note, the proposal, the data from the preliminary analysis, and the final draft of the study report. Their overall support of the study is highly appreciated.

Finally, we appreciate the great contribution of the knowledge management specialist of HIVCore, Ms. Sherry Hutchinson, who coordinated the comments of various reviewers and responses of the authors throughout the various drafts of the study report.
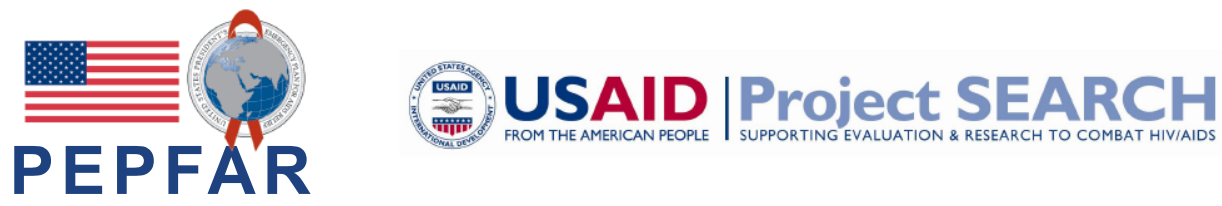

This report was made possible through support provided by the President's Emergency Plan for AIDS Relief and the U.S. Agency for International Development (USAID) via HIVCore, a Task Order funded by USAID under the Project SEARCH indefinite quantity contract (Contract No. AID-OAA-TO-11-00060).

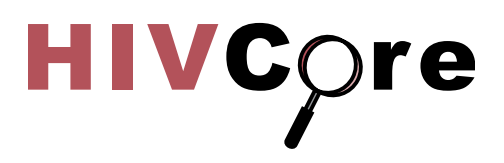

HIVCore improves the efficiency, effectiveness, scale, and quality of HIV treatment, care, and support, and prevention of mother-to-child transmission (PMTCT) programs. The Task Order is led by the Population Council in partnership with Elizabeth Glaser Pediatric AIDS Foundation, Palladium (formerly Futures Group), and the University of Washington.

Published in January 2016. (C2016. The Population Council Inc.

Suggested citation: Kalibala, S., J. Okal, B. Zieman, N. Jani, L. Vu, L.Ssali, J. Birungi, S. Okoboi, J. Wangisi, C. Nabiryo, J. Lyavala, M. Etukoit, L. Buzaalirwa, A. Lubanga, P. Kiyingi, J. Ikapule, S. Nakyeyune, S. Nakibinge, P. Iutung, S. Waliggo, J. Nsamba, Y. Wamanga, J. Kamya, R. Sentongo, and R. Yiga. 2016. "Retrospective review of task-shifting community-based programs supporting ARV treatment and retention in Uganda," HIVCore Final Report. Washington, DC: USAID | Project Search: HIVCore. 


\section{TABLE OF CONTENTS}

ACRONYMS IV IV I I I I I I I I I I I

EXECUTIVE SUMMARY I

$\begin{array}{ll}\text { INTRODUCTION } & 7\end{array}$

Problem statement $\quad 7$

Emerging solution: task-shifting for provision of ART $\quad 7$

Task-shifting strategies 9

STUDY OBJECTIVES $\quad$ I2

METHODOLOGY I 3

$\begin{array}{ll}\text { Study design } & 13\end{array}$

$\begin{array}{ll}\text { Study sites } & 14\end{array}$

Data collection methods and analysis 16

RESULTS $\quad$ 2 I

HIV treatment task-shifting approaches of participant organizations 21

$\begin{array}{ll}\text { Patient-level outcomes } & 31\end{array}$

$\begin{array}{ll}\text { Health systems outcomes } & 38\end{array}$

$\begin{array}{ll}\text { LIMITATIONS } & 44\end{array}$

Overall study limitations $\quad 44$

Limitations of management information system data $\quad 44$

Limitations of costing data $\quad 45$

$\begin{array}{ll}\text { DISCUSSION } & 47\end{array}$

CONCLUSIONS

$\begin{array}{ll}\text { RECOMMENDATIONS } & 52\end{array}$

$\begin{array}{ll}\text { REFERENCES } & 53\end{array}$ 


\section{ACRONYMS}

\begin{tabular}{|c|c|}
\hline AIDS & Acquired immunodeficiency syndrome \\
\hline AHF & AIDS Healthcare Foundation \\
\hline ART & Antiretroviral treatment \\
\hline ARV & Antiretroviral \\
\hline ASO & AIDS service organization \\
\hline CASA & Community ART support agents \\
\hline CDDP & Community-based drug distribution points \\
\hline CHW & Community health worker \\
\hline $\mathrm{CI}$ & Confidence interval \\
\hline CPI & Client-provider interaction \\
\hline HIV & Human immunodeficiency virus \\
\hline HW & Health workers \\
\hline IQR & Interquartile range \\
\hline LHW & Lay health worker \\
\hline LTFU & Loss to follow up \\
\hline MIS & Management information system \\
\hline $\mathrm{MOH}$ & Ministry of Health \\
\hline NGO & Nongovernmental organization \\
\hline OR & Odds ratio \\
\hline PHW & Professional health worker \\
\hline PLHIV & People living with HIV \\
\hline RA & Research assistant \\
\hline RCT & Randomized controlled trial \\
\hline $\mathrm{ROM}$ & Reach Out Mbuya \\
\hline SE & Standard error \\
\hline TASO & The AIDS Support Organization \\
\hline TASO-C & TASO-Central \\
\hline TASO-CDDP & TASO-Community-based drug distribution points \\
\hline TS & Task-shifting \\
\hline UC & Uganda Cares \\
\hline $\mathrm{UC}-\mathrm{MOH}$ & Uganda Cares Ministry of Health \\
\hline UC-S & Uganda Cares stand-alone \\
\hline UGX & Ugandan Shilling \\
\hline USD & United States Dollar \\
\hline VHT & Village health team \\
\hline WHO & World Health Organization \\
\hline
\end{tabular}




\section{EXECUTIVE SUMMARY}

Shortages of human resources for health services have hindered the rollout of antiretroviral treatment (ART) across sub-Saharan Africa. Initial ART rollout models were physician- and hospital-intensive, necessitating task-shifting to quickly expand the capacity of the health workforce to address the burden of ART care services. The World Health Organization (WHO) defines task-shifting as a process of delegation whereby tasks are moved where appropriate to less specialized health workers. Uganda is one of the countries that has adopted task-shifting for ART care. A review of the policy environment for taskshifting was conducted in Uganda in 2009 and it showed high-level support in the Ministry of Health for task-shifting, and a recommendation was made to develop national-level policies and guidelines on taskshifting. However, in developing such guidelines, it would be beneficial to define which tasks can be safely shifted and to which cadre of staff as well as the pre-requisite training needed by these cadres to carry out these tasks. Uganda has a good number of ART task-shifting programs, which should be documented and disseminated widely to guide the policy development process. The purpose of our study, therefore, was to examine examples of task-shifting programs in Uganda with the aim of generating data that could inform the development of task-shifting policies, guidelines, and practices.

\section{OBJECTIVES}

The overall purpose of this study was to contribute to the growing knowledge base about task-shifting by describing the service delivery approaches of three purposively selected major AIDS service organizations (ASOs) in Uganda that have adopted task-shifting: Kitovu Mobile, Uganda Cares (UC), and The AIDS Support Organization (TASO).

The specific study objectives were to:

1. Provide a description of task-shifting approaches in three Ugandan HIV treatment organizations.

2. Document the following patient-level outcomes: knowledge about ARVs, patient recall of interaction with providers and level of satisfaction, and ART adherence and retention among patients accessing services in these three organizations.

3. Document the following health system outcomes: quality of care, time spent by patients, and cost per patient visit in these three organizations.

\section{METHODOLOGY}

Several approaches were used in responding to the three study objectives:

- Objective 1 was addressed using existing program reports and qualitative interviews with key informants and providers from these three organizations.

- Objective 2 and part of Objective 3 (quality of care, time spent by patients) were addressed using quantitative cross-sectional surveys (time-motion study, exit interviews, and client-provider interaction observations) with patients accessing services at these three organizations, and a retrospective review of patient registers. 
- Objective 3 (cost per visit) was addressed by a retrospective review of accounting data.

ART task-shifting can take place at a central health facility, e.g., when a nurse at a central clinic is delegated authority to order or prescribe the refill of ARV supply for stable ART patients without requiring a doctor's prescription. On the other hand task-shifting can take place by shifting ART care from the central health facility to decentralized clinics, e.g., community outreach settings. Task-shifting also varies by the type of task being delegated, e.g., a highly clinical task like initiation of ART or a less clinical task like patient triage. Thus to completely understand the type of task-shifting being referred to we need to describe at least three dimensions: a) by place—a decentralized clinic can be considered to be more task-shifted than a central one; b) by provider-delegation from a doctor to a lay health worker can be considered to be more task-shifted than from a doctor to a clinical officer; and c) by task-delegation of ART initiation can be considered to be more task-shifted than delegation of patient triage (Figure 1). For the purposes of this report, we defined highly task-shifted clinics as clinics providing ART care at relatively more decentralized sites, often using lower level health workers with more clinical tasks being delegated, and minimally task-shifted clinics as those that provide ART care at relatively more central health facilities using higher level health workers and minimizing the delegation of the more clinical tasks. This definition allows different service delivery models to be located at varying points on a spectrum from highly task-shifted to minimally task-shifted depending on the three factors: place, provider, and task.

\section{KEY FINDINGS}

\section{Description of task-shifting approaches of the three ASOs}

Kitovu Mobile: Kitovu Mobile delivers its ART services through task-shifting using non-facility-based community locations and does not have a central clinic. Currently Kitovu Mobile has 111 community outreach sites delivering ART care. The health workers travel by a project vehicle to meet groups of patients at a community location such as a church, the verandah of a home, or under a tree. Thus all the Kitovu Mobile clinics are in a highly task-shifted model.

Uganda Cares: UC operates two types of ART task-shifting models: 1) Uganda Cares stand-alone (UC-S) clinics which are located at UC-owned centers and 2) Uganda Cares MOH (UC-MOH) which provides drugs and other supplies to Ministry of Health $(\mathrm{MOH})$ staff to provide HIV care at $\mathrm{MOH}$ health facilities. Both UC models are considered minimally task-shifted.

The AIDS Support Organization: TASO delivers HIV care through its 11 service delivery centers. Each TASO center has two types of task-shifting models: 1) central, minimally task-shifted clinicsTASO-Central (TASO-C) and 2) about 30 highly task-shifted outreach clinics per TASO center-the community-based drug distribution points (TASO-CDDPs). Patients are initiated on ART at the TASO-C clinics and when they stabilize on ART they are referred to a TASO-CDDP of their choice, where they receive ARV refills on an ongoing basis from a TASO counselor assisted by clients that TASO has identified as "expert clients." CDDPs are conducted on a specific day every other month in a community location such as a place of worship or under a tree. On average there are 30 patients per CDDP. ART patients attending TASO-CDDPs who require a clinical review are referred back to TASO-C for that review, after which they return to the TASO-CDDP to resume ongoing ARV refill. Thus, no TASO patients are categorically CDDP or central clinic patients, but rather each patient receives some services at the CDDP and the central clinic. 
Shifting of tasks: The health worker (HW) interviews showed that more tasks tended to be delegated from the health workers at the apex of the medical profession-doctors and clinical officers-than were delegated to them. For the mid-level health workers—nurses and counselors- there was similar likelihood of tasks being delegated from and to them. Tasks were more likely to be delegated to than from providers at the bottom of the rank-expert clients and HIV medics. For example, the facility audits showed that the task of nurses to perform pre-clinical care was delegated to lay health workers (LHWs) in all the models while nurses were having the tasks of clinical assessment, counseling, and dispensing being delegated to them in some models. Similarly, at TASO-CDDP, facility audits showed that counseling was delegated from counselors to LHWs while counselors got the tasks of dispensing drugs delegated to them.

Training: The majority of professional health workers (PHWs) and LHWs who were delivering ART in all models reported receiving training in ART care-in Kitovu Mobile, 82 percent (9/11) PHWs and 100 percent (26/26) of LHWs; in UC, 100 percent (50/50) of PHWs and 93 percent (39/42) of LHWs; and in TASO, 83 percent (74/89) of PHWs and 84 percent (31/37) of LHWs. About half or more of LHWs reported having been trained in ART counseling and health education, which perhaps reflects the two tasks that were most delegated to them. For example, the following proportions of LHWs were trained in counseling: in Kitovu Mobile, 88 percent (23/26); in UC, 71 percent (30/42) and in TASO, 49 percent (18/37). However, half or less of PHWs reported receiving training in the specific skills required of them-prescribing and dispensing. For example, the following proportions of PHWs were trained in prescribing: in Kitovu Mobile, 36 percent (4/11); in UC, 50 percent (25/50); and in TASO, 39 percent $(35 / 89)$.

\section{Patient-level outcomes}

Client exit interviews showed that client knowledge ${ }^{1}$ about ARVs was low (32-35 percent) in all three ASOs. However, patient recall of receiving key elements of an ARV discussion with the provider in the clinic that day varied from 59 percent to 66 percent and was significantly higher in the facilitybased clinics compared to community-based settings $(\mathrm{p}<0.05)$. Client satisfaction with the services received that day was over 90 percent in all models but was significantly higher in the facility-based clinics compared to community-based settings $(\mathrm{p}<0.05)$. This difference in recall, while significant, was programmatically marginal at 7 percentage points, and it is important to note that client satisfaction was quite high in both community-based and facility-based clinics.

Regarding ARV adherence while in ART care-as measured by patient medication refill records_patient histories from the three ASOs after 24 months of follow-up showed 55 percent of patients had received sufficient ARV medication to cover at least 95 percent of their expected treatment. Retention in ART care was measured by compiling patient histories for patients who initiated ART between July 1 and December 31, 2010 and determining what proportion was retained in care 24-30 months later, at December 31, 2012. At 24 months, of the 2,223 patients from all sites, 1,667 patients ( 75 percent) were actively retained in care, 16 patients ( 0.7 percent) had transferred to another facility, and six patients (0.3 percent) had died. The remaining 24 percent of patients (534) had defaulted on their care without resuming treatment by December 31, 2012, and had no final outcome documented in their records; all

${ }^{1}$ Regarding $A R V$ knowledge, the respondents were asked about the following aspects: benefits of ARVs, side effects of $A R V s$, adherence requirements of ARVs, and what happens if one is not adhering to ARVs 
were deemed lost to follow-up. However, the quality and completeness of final patient outcome records varied by organization and facility. Therefore, it was not possible to ascertain whether any patients who were lost to follow-up may have actually died or transferred to another facility. From the perspective of improving drug adherence, facilities should consider that any patients who default need support until the patient resumes care or an ultimate outcome can be documented.

Only TASO provided data from ART services delivered to patients at a central facility and through a community-based care model: the TASO-CDDPs. Bivariate analysis showed that stable TASO patients who received any services through a CDDP were significantly more likely to be retained at 12 months (OR: 16.3; 95\% CI: 10.3-25.9) and 18 months (OR: 13.0; 95\% CI: 8.5-20.0) than patients who attended no CDDP-based visits. However these estimates may be influenced by patient treatment preferences, or by patients who had received no CDDP-based services because they were not sufficiently stable after their first three refill visits to attend.

\section{Health systems outcomes}

To examine the quality of care under task-shifted services, we observed the performance of key tasks performed by various cadres of HWs during sessions of client-provider interaction (CPI). The results show that provider performance in the clinical assessment ${ }^{2}$ of the patients did not differ by level of taskshifting-66 percent at community-based and 74 percent at facility based $(\mathrm{p}>.05)$. There was also no significant difference in adherence counseling- 42 percent at community-based vs 55 percent at facilitybased $(\mathrm{p}>.05)$ and for counseling about side-effects-30 percent at community-based vs 42 percent at facility-based $(\mathrm{p}>.05)$. However, there was a significant difference in addressing patients' complaints: 70 percent at community-based vs 89 percent at facility-based $(\mathrm{p}<.01)$. Therefore, while the performance of providers at community-based settings was similar to those at facility-based clinics in most tasks observed, provider performance in addressing the patients' complaints was significantly lower at the communitybased settings. At Uganda Cares, there was no difference in the performance of these tasks between HIV medics (lay health workers) on one hand and professional health workers (doctors, clinical officers, and nurses) on the other.

We also conducted time-motion studies to examine the time efficiency of these task-shifted services. The median time spent at the clinic was 48 minutes at UC-S, 128 minutes at UC-MOH, 200 minutes at TASO-CDDP, 221 minutes at TASO-C, and 225 minutes at Kitovu Mobile. Of this time, the time spent waiting ranged from 33 minutes at UC-S to 195 minutes at Kitovu Mobile. The contact time with clinic staff appears to be within the same order of magnitude across the five models (15 to 29 minutes). However, the percentage of time spent in contact with the provider was highest at UC-S (37.5 percent) and lowest at Kitovu Mobile (6.7 percent). Hence, there was poor time efficiency at the community-based settings compared to facility-based clinics.

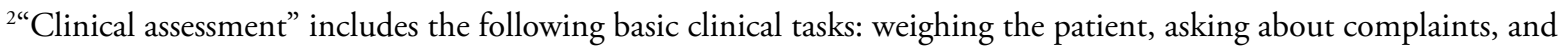
exploring issues mentioned by the client. 


\section{Costing}

Cost varied across sites within each organization as well as across the three organizations. In addition, the number of annual ART visits was more frequent in rural areas and among Kitovu Mobile (community distribution model), which played a major part in the overall annual ART cost. The annual cost per client was $\$ 404$ for Kitovu Mobile, \$332 for TASO, and \$257 for UC, which were lower than the previous analyses in Uganda and the region (Menzies et al. 2011). ART drugs accounted for the majority of the total cost, followed by personnel and operational costs.

\section{CONCLUSIONS AND RECOMMENDATIONS}

This study showed that in the three ASOs, almost all key tasks in ART, including determining eligibility, initiating ART, and dispensing ARVs, can be feasibly shifted to lower level cadres or lower level facilities. While WHO has defined task-shifting using one dimension-the personnel involved in the taskshifting - the data from our study adds two other dimensions that could be used for a more descriptive definition - type of tasks delegated and type of facility to which ART services are delegated. The increased analytic robustness of this approach leads us to believe that task-shifted services should be assessed across all three dimensions: the type of cadre, the type of facility, and the type of task.

Our study has identified training gaps in specific areas of competence among PHWs and LHWs currently providing ART in task-shifted services. Therefore, when rolling out task-shifting in Uganda, it will be important to conduct training needs assessments of both the PHWs and LHWs and provide them with the required training in order to ensure competence and quality of care in task-shifted services.

The data showed that some of the models demonstrated efficient use of patient time. The reasons for the efficient use of patient time in models such as UC-S should be further explored and shared widely as best practices that can strengthen other task-shifting models.

Our study provides the most recent available costing data from the three largest HIV service organizations, representing three different ART service delivery models in Uganda. Unit costs, cost distribution, and resource utilization varied widely across the three sites and models, suggesting the potential for efficiency gains in ART service delivery. In particular, HIV programs in Uganda may save costs by reducing the number of annual ART visits to the national standard (four ART visits a year on average). Our findings of lower annual ART costs compared to previous analyses in Uganda and the region indicate that we may be able to treat more people with the same or even fewer resources.

As referenced in the main body of the report, there is a growing body of literature on ART task-shifting. Indeed, to date at least three meta-analyses have been carried out on the subject. In summary, the studies point toward a general conclusion that task-shifting in any of the three dimensions-by type of provider, by level of facility, and by complexity of task delegated-mostly seems to result in the same level of outcomes when compared to the minimally task-shifted ideal of a doctor providing ART initiation and maintenance in a hospital. However, some studies suggest better results from task-shifted ART care while others suggest poorer results from task-shifted ART care. Our study has shown similar conclusions.

When we compared task-shifting by facility level we found that community-based settings were similar to facility-based clinics with regard to the following indicators: ARV knowledge of clients; and provider performance in clinical assessment of patients, adherence counseling, and counseling about side effects. Our data also showed that community-based settings had better retention in ART. However, the data also 
suggest that community-based settings performed less well than facility-based settings on the following indicators: client recall of discussions with provider, overall client satisfaction, provider performance in addressing client's complaints, and time efficiency. Thus our study has shown that in the three ASOs almost all key tasks in ART including determining eligibility, initiating ART, and dispensing ARVs can be feasibly shifted to lower level cadres or lower level facilities mostly with non-inferior outcomes compared to non-task-shifted ART care. Findings from this study provide important insight on costs and outcomes associated with task-shifting in facility-based versus outreach/community-based service environments

We thus make the following recommendations:

- Consideration should be made to expand the description of task-shifted services along more than one dimension: the type of cadre, the type of facility, and the type of task.

- When rolling out task-shifting in Uganda it will be important to conduct a training needs assessment of both the PHWs and LHWs and provide them with the required training in order to ensure competence and quality of care in task-shifted services. Given the likelihood of staff attrition, recruitment of new staff, and changes in guidelines, special attention should be paid to continued medical education for all cadres of staff involved in ART care.

- In our study we have shown that quality of care did not differ between HIV medics and other health workers (doctors, clinical officer, and nurses) working at the same type of clinic (UC). This suggests that the HIV medics are a cadre worth exploring for the scale-up of task-shifting.

- The reasons for the efficient use of patient time in models such as UC-S should be further explored and shared widely as promising practices that can strengthen other task-shifting models.

- Kitovu Mobile should examine current ART dispensing (or documentation) practices as, on average, the documentation shows that clients are receiving insufficient drugs to cover the interval between refills, and only 19 percent of clients achieved 95 percent drug coverage during their time in care.

- Given their low levels of drug coverage yet high levels of retention, Kitovu Mobile should consider examining client viral loads to proactively identify any systemic concerns regarding viral suppression and drug resistance.

- Future programs might benefit from examining the distribution of costs among the different elements of a task-shifted program, in order to identify care packages that deliver the highest quality of care at the lowest cost.

- The collection of costing data to measure unit costs, cost-effectiveness, and cost-efficiency remains critical. ART service delivery sites in country would benefit from implementing a standardized cost data-capture tool or M\&E system that allows for comparison across sites. 


\section{INTRODUCTION}

\section{PROBLEM STATEMENT}

Uganda was one of the first countries to be heavily impacted by the HIV/AIDS epidemic since the first AIDS case was identified in the early 1980s. The country is widely considered to be an HIV/AIDS control success story having reduced its prevalence rate from 18.5 percent of adults aged 15-49 years in 1992 to 6.4 percent in 2004 (Uganda AIDS Commission 2014). However, it is estimated that 140,908 new infections occurred in 2013, and the HIV prevalence in the country rose to a national average of 7.3 percent $(\mathrm{MOH} 2011)$. The disease has created a severe and unsustainable burden on the already scarce health-sector resources as funds are tapped from other sectors for HIV prevention and AIDS care and treatment services. Approximately 1.1 million Ugandans have died from AIDS (MOH and ORC Macro 2006). Sexual transmission continues to contribute 76 percent of new HIV infections, while motherto-child transmission accounts for 22 percent of infections in the country (Uganda AIDS Commission 2011).

At the end of 2013 it was estimated that 1.6 million people were living with HIV in Uganda (Uganda AIDS Commission 2014); and 69.4 percent of those eligible for antiretrovirals (ARVs), using the 2010 World Health Organization (WHO) guidelines, were on antiretroviral treatment (ART). However, using the 2013 WHO guidelines, which have a lower cut-off threshold, those on ART would represent only 40 percent of those eligible for treatment (Uganda AIDS Commission 2014). The provision of ART has enabled an entire generation of the country's HIV infected population to live longer, more productive, and healthier lives. However the advent of these lifesaving drugs comes with challenges to health care systems of developing countries such as Uganda. Healthcare worker shortage is a major constraint to ARV care in Uganda. While medical doctors are traditionally considered the most qualified health care providers to determine patient eligibility for ART and initiate ART care, it is estimated that there is only one doctor for every 22,000 patients in Uganda, which amounts to an 80 percent overall health worker (HW) deficit (WHO 2007).

\section{EMERGING SOLUTION:TASK-SHIFTING FOR PROVISION OF ART}

Shortages of human resources for health have hindered the rollout of ART across sub-Saharan Africa. Current ART rollout models are physician and hospital intensive, highlighting that alternative, simplified models are needed to quickly expand the capacity of the current health workforce. In 2008 WHO developed guidance on task-shifting to address HW shortage and enhance access to ARV care in countries such as Uganda. WHO defines task-shifting as a process of delegation whereby tasks are moved, where appropriate, to less specialized HWs (WHO 2008).

To date at least three meta-analyses have been carried out on the subject of ART task-shifting. One meta-analysis published in 2010 (Callaghan, Ford, and Schneider 2010) found articles covering three dimensions: tasks_-initiation and maintenance of ART; providers—-delegation from doctors to nurses or 
to other non-physician providers; and level of facility-decentralized vs. central clinics. They reported that among the studies they reviewed there was evidence of increased access to and retention in ART resulting from task-shifting, the quality of care was equal or better with task-shifting, and task-shifting was costeffective. However, they observed that in one study task-shifted providers who had not received training since their pre-service training were more likely to make errors, and in another study LHWs were worse than PHWs at identifying clients eligible for ART. They highlighted the need for programs to identify which tasks should and should not be delegated to some LHWs and the need for continued training of providers.

Another meta-analysis published in 2013 (Kredo et al. 2013) reviewed task-shifting studies comparing central vs. decentralized service sites. They grouped the studies into those examining "partial decentralization," where ART was initiated at a hospital and maintained at a health center; "full decentralization," where ART was initiated and maintained at a health center; and "ART in the community," where ART was initiated in a hospital or health center and maintained in the community. The last category, "ART in the community," is perhaps similar to the TASO model in our study. They concluded that partial decentralization and full decentralization had reduced loss to follow-up compared with hospital initiation and continuation. They also reported no difference in loss to follow-up, virological and immunological outcomes, and death between any of the levels of decentralization.

A third meta-analysis published in 2014 (Kredo et al. 2014) focused on task-shifting studies comparing type of provider and type of task delegated. In one group of studies comparison was made between doctors and nurses or clinical officers (COs) for two tasks: initiating and maintaining ART. They reported mixed results. The randomized controlled trials showed that there was no difference in mortality and that there was less loss to follow-up with the nurses/COs compared with doctors. However, the cohort data showed no difference in loss to follow-up but an increased risk of death in the nurses/COs arm. The second group of studies compared doctors to nurses/COs for one task: maintenance of ART. Trials data showed that there was no difference between arms in death and loss to follow-up. On the other hand, cohort data showed that there were fewer deaths and fewer losses to follow-up in the nurses/COs arm compared to doctors. The third group of studies compared doctors to LHWs for one task: maintenance of ART, and for type of facility: doctors delivering ART in hospitals and LHWs delivering ART in homes. There were no cohort studies in this group of studies but the trials data showed no difference in death and loss to follow-up between the two arms.

In response to the growing evidence base, Uganda extended its human resource capacity for delivering HIV/AIDS services by creating a range of non-professional HWs who are trained to provide specific aspects of ARV care. Consequently, the burden of patient care began to shift to lower level HWs such as nurses, pharmacists, expert clients, and community health workers (CHWs). Ugandan nurses assumed a range of tasks that formerly fell within the purview of doctors including managing opportunistic infections among people living with HIV (PLHIV), diagnosing tuberculosis based on sputum results, deciding whether PLHIV have medical eligibility for ART, and managing side effects for people on ART (WHO 2007). Consequently the delegation of responsibility goes further, as tasks that formerly fell under the responsibility of nurses have been shifted to CHWs who have received job-related training, but do not necessarily possess professional qualifications.

An increasing number of studies have highlighted the impact of ART task-shifting programs in Uganda. One study examined ART task-shifting in $12 \mathrm{MOH}$ health facilities from three regions in Uganda where 
the performance of nurses and clinical officers was compared to doctors in the task of deciding to initiate clients on ART and found concurrence between these three groups of professional health workers (Vasan et al. 2009). In addition, shifting ART care tasks from doctors by establishing nurses-only visits and pharmacy refills visits was reported to improve client time efficiency at the infectious disease institute in Mulago Hospital, Kampala (Castelnuovo et al. 2009). Delegating ART care responsibilities further down to community levels has also been tested. In an evaluation of a community-based ART program in western Uganda, it was reported that there were improvements in both clinical outcomes and quality of life, demonstrating that positive outcomes can be achieved in community-based ART programs (Alibhai 2009).

Shifting ART tasks from doctors to less costly providers could save money for the ARV program. An analysis of the cost-saving aspect of task-shifting in ART care in Uganda was carried out and it was concluded that task-shifting results in substantial cost and physician personnel savings in ART follow-up in Uganda and can contribute to mitigating the health worker crisis (Babigumira et al. 2009).

For a new innovative care approach such as task-shifting it is vital to have evidence-based continued quality improvement as it is rolled out. Reach-Out Mbuya (ROM) is a community-based ART program in Uganda that implements and continuously evaluates task-shifting in its service delivery. ROM operates a nurse-led ART care approach which was evaluated for time efficiency in 2008 in comparison to doctorled care in Mulago Hospital. It was reported that the nurse-clinicians at ROM spent twice the time with patients compared with the doctors at Mulago (Wanyenze et al. 2010). In a further refinement of the client flow at ROM in 2008 and 2009, it was possible to document improved time efficiency (Alamo et al. 2013). Another approach used by ROM is to delegate to LHWs the task of monitoring ART patients for side effects through home visits. A review of this program revealed various challenges that need to be addressed including work overload and the need to triage patients so as to prioritize visiting unstable patients over patients who have stabilized on ART (Alwano et al. 2012). The experience of ROM shows the need to continue refining task-shifting programs using rigorous evaluations to inform decision makers.

\section{TASK-SHIFTING STRATEGIES}

Task-shifting can be examined in at least three dimensions: the tasks that are shifted, the providers between whom the tasks are shifted, and shifting of service delivery venues from higher level, centrally located, health facilities to lower level, decentralized sites. In the 2008 WHO guidelines (WHO 2008) task-shifting is characterized using one dimension-the personnel. Our study seeks to expand the above definition by characterizing task-shifting along two additional dimensions - type of tasks delegated, and type of venue to which ART services are delegated. Figure 1 illustrates this three-dimensional nature of task-shifting. In the top-left corner is the minimally task-shifted situation, for example a hospital where doctors initiate patients on ART and keep seeing them routinely for clinical monitoring and ARV refill. It is shown that the amount of task-shifting can be increased in three planes: a) decreasing provider qualifications from doctor to clinical officer to nurse and lay health worker (LHW); b) decreasing facility level from hospital to health center and community setting; and c) increasing complexity of task delegated from patient triage to ARV refill to initiation of ART. Maximizing task-shifting in all three dimensions 


\section{Figure I:Three-dimensional framework of task-shifting}

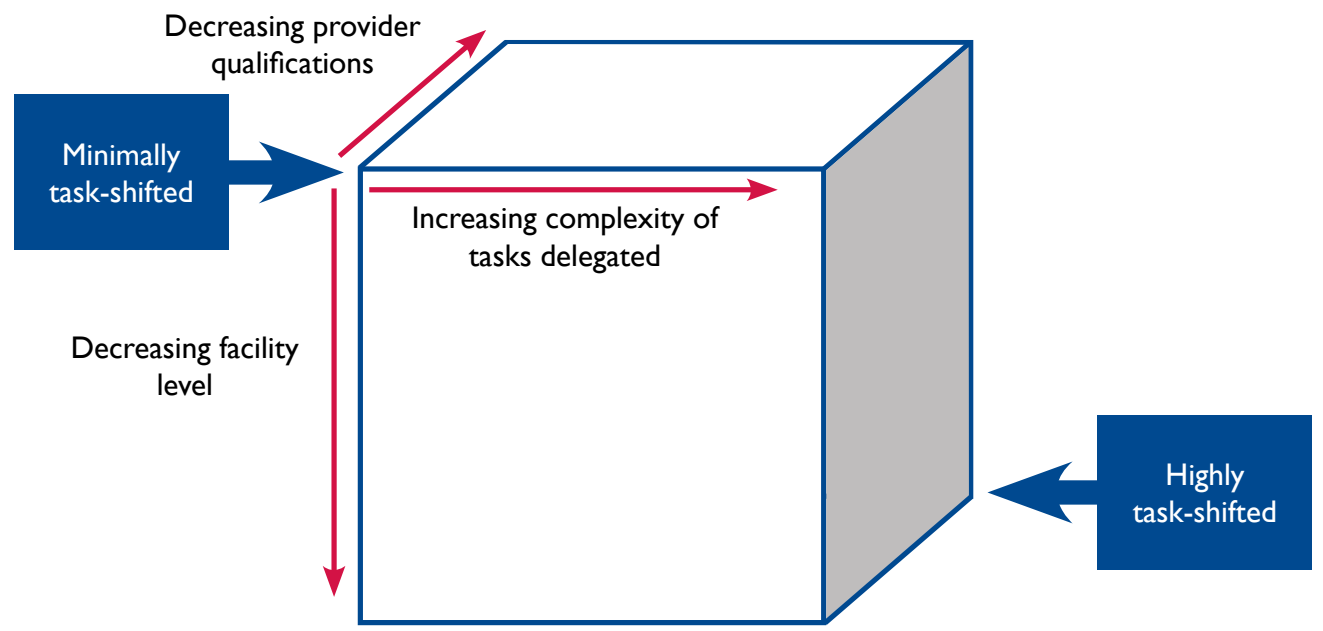

can result in a highly task-shifted situation where, for example, trained LHWs are initiating clients on ART in a community setting.

\section{Tasks that can be shifted}

Task-shifting strategies are relative to which tasks are delegated and are also often context specific. Callaghan and colleagues (Callaghan, Ford, and Schneider 2010) report that the tasks performed by the lower cadres include triage at the clinic, adherence support, review of signs and symptoms of drug toxicity or disease progression, ART medication refill, and HIV testing and counseling of family members.

Examples of ART tasks that can be shifted in various task-shifting models include:

- ART initiation: Review of the patient's history, conducting psychosocial assessments, conducting a physical exam, interpreting relevant laboratory test results to make a decision about whether the patient is eligible for ARVs, and writing the first prescription of ARVs for the patient.

- ARV refill: Review of the ART patient's adherence and any side effects, conducting a physical exam, and interpreting any laboratory test results to make a decision to prescribe or order a re-supply of ARVs for the patient.

- Dispensing of ARVs: Interpretation of a prescription or order of ARVs for a patient, counting or obtaining the correct quantity and types of the prescribed or ordered ARV drugs, handing the ARVs to the patient as well as explaining the usage of the drugs to the patient and ensuring understanding of instructions.

\section{Task-shifting among providers}

Task-shifting involves the delegation of tasks from a higher level standard provider to a lower level member of the ART team. Typically the ART team comprises several categories of personnel including (WHO 2008; Zachariaha et al. 2009):

- Clinicians (doctors and clinical officers) whose standard roles are to carry out patient consultation, 
manage opportunistic infections, clinical staging, and ART initiation.

- Nursing staff (nurses and nursing assistants) whose standard roles are triage, supervising CHWs, and supporting doctors by preparing individuals for ART and monitoring ART recipients.

- Counseling staff (counselors and counseling assistants) whose standard role is to provide adherence counseling.

- Pharmacy staff whose standard role is dispensing medicines.

- Lay providers (expert clients, CHWs, village health teams, etc.) who are usually trained by organizations to carry out specific aspects of care and support including triage, health talks, adherence counseling, and emotional support.

- Other providers including laboratory staff, family members, etc.

\section{Task-shifting between health care facilities}

ART task-shifting can take place at a central health facility, e.g., a national or district hospital which normally provides HIV treatment. One example is when a nurse at such a central clinic is delegated authority to order or prescribe the refill of ARV supply for stable ART patients without requiring a doctor's prescription. On the other hand task-shifting can take place by shifting ARV care from the central hospital to decentralized clinics, thus enhancing ART access for patients in areas distant from central clinics (Callaghan, Ford, and Schneider 2010). The decentralized site could be a peripheral health center, a community center, a place of prayer, a patient's home, or just under a tree. In addition to shifting the venue, the decentralized clinics also often shift the personnel conducting some of the tasks, for example, delegation of ARV refills prescription from a doctor to a nurse. In this sense the decentralized clinics can be considered to be highly task-shifted compared to central clinics since the decentralized clinics have shifted the venue and the personnel while the central clinics may only shift the personnel (minimally task-shifted).

\section{Need for further exploration of task-shifting in ART care}

Task-shifting in the context of ARV care has been taking place in Uganda and other sub-Saharan African countries as a strategy to enhance access to ARV care. While WHO has developed global guidance (WHO 2007) on task-shifting, there is need for countries such as Uganda to develop national policies and guidelines to guide the roll-out of task-shifting and best practices. To this effect, a review of the policy environment for task-shifting was conducted in Uganda in 2009 that found a high level support for task-shifting and made recommendations to develop national level policies and guidelines on task-shifting (Dambisya 2012). However, in developing such guidelines it would be beneficial to define which tasks can be safely shifted and to which cadre of staff (Callaghan, Ford, and Schneider 2010), as well as the prerequisite training needed (Lutalo et al. 2009) by these cadres to carry out these tasks. While the literature about ART task-shifting in Uganda is growing, Uganda still has a good number of ART task-shifting programs that have not been adequately documented or disseminated (East, Central, and Southern African Health Community 2010). The purpose of our study, therefore, was to examine examples of task-shifting programs in Uganda with the aim of generating data that could inform the development of task-shifting policies, guidelines, and practices. 


\section{STUDY OBJECTIVES}

The overall purpose of this study was to add to the growing body of knowledge about task-shifting for ART and contribute to the strengthening of national policy and development of service delivery guidelines that would indicate how task-shifting should be carried out, and the expected benefits of taskshifting to the patient and the health system.

The specific study objectives were to:

1. Provide a description of task-shifting approaches in three Ugandan HIV treatment organizations.

2. Document the following patient-level outcomes: knowledge about ARVs, patient recall of interaction with providers and level of satisfaction, and ART adherence and retention among patients accessing services in these three organizations.

3. Document the following health system outcomes: quality of care, time spent by patients, and cost per patient visit in these three organizations. 


\section{METHODOLOGY}

\section{STUDY DESIGN}

Several approaches were used in responding to the three study objectives, including quantitative cross sectional surveys, qualitative interviews, observation of service delivery, and a retrospective review of service statistics and accounting data. The three AIDS service organizations (ASOs) included in the study, started delivering ART in the 2002 (Uganda Cares), 2003 (TASO), and 2006 (Kitovu Mobile). They each sooner or later applied task-shifting strategies in 2004 (Uganda Cares), 2004 (TASO), and 2006 (Kitovu Mobile). The retrospective review of service statistics covered the period July 2010 to December 2012, the retrospective review of accounts data covered the period January to December 2012, and the surveys were conducted between June and September 2013.

Specifically:

- Objective 1 (task-shifting approaches) was answered using existing program reports and qualitative interviews with key informants and providers in participating organizations.

- Objective 2 and part of Objective 3 (quality of care, time spent by patients) were answered using quantitative cross sectional surveys (time-motion study, exit interviews) with patients accessing services at the three organizations and a retrospective review of service statistics.

- Objective 3 (cost per visit) was answered through a retrospective review of accounting data.

Table 1 summarizes the different data collection methods corresponding to the three study objectives.

Table I Study methodology overview

\begin{tabular}{|c|c|c|}
\hline Output & Data collection & Analysis \\
\hline \multirow{3}{*}{$\begin{array}{l}\text { Description of task-shifting } \\
\text { approaches }\end{array}$} & Interviews with HWs & Descriptive statistics \\
\hline & Facility audits & Descriptive statistics \\
\hline & Review of program reports & $\begin{array}{l}\text { Extraction of main themes about task- } \\
\text { shifting }\end{array}$ \\
\hline \multirow[t]{3}{*}{ Document patient outcomes } & Client exit interviews & Descriptive statistics \\
\hline & $\begin{array}{l}\text { Retrospective review of } \\
\text { service statistics }\end{array}$ & $\begin{array}{l}\text { Adherence: Proportion of days covered } \\
\text { by drugs picked up }\end{array}$ \\
\hline & & $\begin{array}{l}\text { Retention: Proportion of cohort still in } \\
\text { program at end date }\end{array}$ \\
\hline \multirow[t]{3}{*}{ Document health systems outcomes } & CPI observations & Descriptive statistics \\
\hline & Time-motion study & Descriptive statistics \\
\hline & $\begin{array}{l}\text { Retrospective review of accounting } \\
\text { data }\end{array}$ & Descriptive statistics \\
\hline
\end{tabular}




\section{STUDY SITES}

We purposively selected three major organizations that provide HIV and ART services to PLHIV in Uganda using a task-shifting approach: Kitovu Mobile, Uganda Cares (UC), and The AIDS Support Organization (TASO). The three organizations were selected based on stakeholder recommendations and the current task-shifting situation in Uganda. These three AIDS-service organizations (ASOs) differ in size and service delivery approaches. Within each of these three ASOs, the selection of study clinics was purposive, aimed to ensure a fair distribution across: 1) the geographical area of operation; 2) high- vs. low-patient volume clinics, and 3) highly task-shifted versus minimally task-shifted clinics. For the purposes of this report we defined highly task-shifted clinics as clinics providing ART care at community outreach sites and minimally task-shifted clinics as those that provide ART care at health facilities. Overall, 28 facilities were selected across the three organizations. The distribution of the clinics is summarized in Table 2, and details are provided in Figure 2.

\section{Table 2 Summary of clinic selection}

\begin{tabular}{|lcccccc|}
\hline & $\begin{array}{c}\text { Patients } \\
\text { currently } \\
\text { on ART in } \\
\text { June 20 I4 }\end{array}$ & $\begin{array}{c}\text { Geographical } \\
\text { area of } \\
\text { operation }\end{array}$ & $\begin{array}{c}\text { Geographical } \\
\text { area selected } \\
\text { for the study }\end{array}$ & $\begin{array}{c}\text { Highly } \\
\text { task-shifted } \\
\text { clinics (High } \\
\text { volume) }\end{array}$ & $\begin{array}{c}\text { Highly } \\
\text { task-shifted } \\
\text { clinics (Low } \\
\text { volume) }\end{array}$ & $\begin{array}{c}\text { Minimally } \\
\text { task-shifted } \\
\text { clinics }\end{array}$ \\
\hline $\begin{array}{l}\text { Kitovu } \\
\text { Mobile }\end{array}$ & I,439 & $\begin{array}{c}\text { 6 districts in } \\
\text { I region }\end{array}$ & I region & 2 & 2 & 0 \\
$\begin{array}{l}\text { Uganda } \\
\text { Cares }\end{array}$ & 50,039 & $\begin{array}{c}\text { I3 districts in } \\
\text { 4 regions } \\
\text { TASO }\end{array}$ & II centers in \\
I0 regions & 58,584 & 5 regions & 5 & 0 & 9 \\
\hline
\end{tabular}

\section{Kitovu Mobile}

Kitovu Mobile AIDS Organization commonly known as Kitovu Mobile is a faith-based organization under the Catholic Diocese of Masaka, Uganda. The organization is among the pioneer programs for home-based care in Uganda. It has provided HIV care and support since 1987 using a community outreach model, thus its name-Kitovu Mobile. Kitovu Mobile has no central ART clinic. In this study we categorized Kitovu Mobile as being a highly task-shifted model since ART service delivery was decentralized to community outreach settings. Kitovu Mobile conducts its operations at 111 community outreach sites located in six districts in the Central-1 region of Uganda. Within these six districts of Kitovu Mobile operations, two districts were selected and within each district two clinics were selected: one low volume and one high volume, resulting in a total of four clinics. The following clinics were purposively selected by the study team based on information provided by the ASO to ensure a reasonable, fair distribution across districts and low and high-volume clinics: Bukulura, Kabuwoko, Kyazanga, and Mbirizi.

\section{Uganda Cares (UC)}

Uganda Cares (UC) started providing ART care at its first center in Masaka, Uganda in February 2002 (Uganda Cares 2003) and applied its HIV medic task-shifting program in 2004 (Hiner and Musokwa 2007). UC provides ART using two models. In one model the clinics are entirely owned and managed 
Figure 2 Map of Uganda showing HIV prevalence in $201 \mathrm{I}$ by region and location of TASO, Kitovu Mobile, and UC

\author{
(1)
}

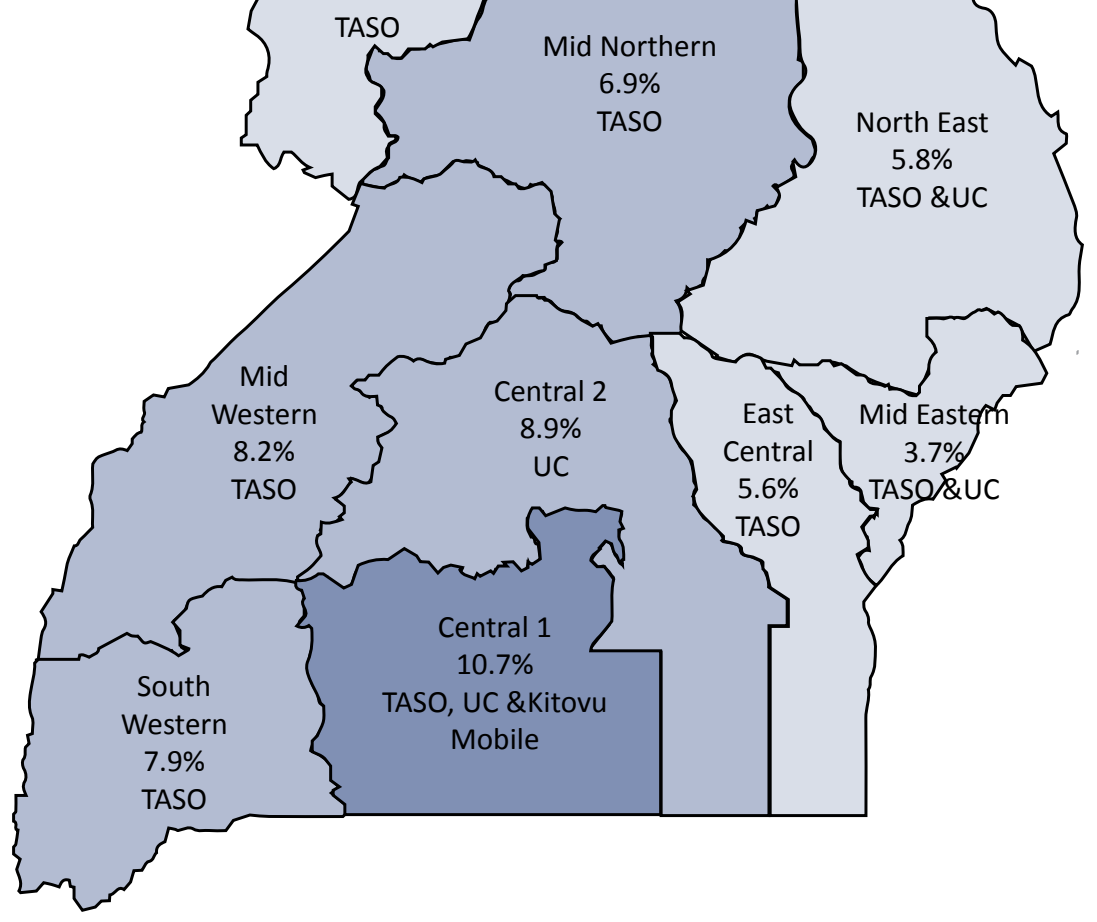

by the ASO_Uganda Cares Stand-alone (UC-S) clinics. In the second model UC provides drugs and supervision to existing $\mathrm{MOH}$ clinics that provide ART using $\mathrm{MOH}$ personnel and premises-Uganda Cares $\mathrm{MOH}$ (UC-MOH) clinics. There are no referral links between these two UC models. We consider both UC models to be minimally task-shifted in that the ART services are provided at health facility clinics as opposed to community outreach programs. Currently, UC is operating 29 clinics in 13 districts in the North-East, Mid-East, Central-1, and Central-2 regions of the country. Among the four regions of operation the study team purposively selected the following clinics with the aim of ensuring a reasonably fair distribution across regions of Uganda and UC-S vs. UC-MOH: three UC-S clinics: 1) Soroti (NorthEast region), 2) Nakawa, and; 3) Balikuddembe (Central-2 region); six UC-MOH clinics: 1) Maddu, 2) Rakai, 3) Lyantonde, and 4) Kalisizo (all four in Central-2 region); and 5) Mulanda and 6) Nagongera (both in Mid-East region).

\title{
The AIDS Support Organization (TASO)
}

TASO is a large ASO that provides ART using clinics that are entirely managed by the ASO. TASO started delivering ART care in May 2003 at its Tororo Center; in early 2004 TASO rolled out ART care at all its centers. In mid-2004 TASO started its first task-shifting strategy when it trained and used lay health workers to deliver ARV refills to client's homes (Stilwell 2007). Currently, ART is provided at TASO Central (TASO-C) clinics or at TASO Community Drug Distribution Points (TASO-CDDP) clinics. The two TASO models have a two-way referral link in that patients who are stable on ART are 
referred from TASO-C to TASO-CDDP for ongoing ARV refills and CDDP patients who are unstable or need a medical review are referred back to TASO-C. In this study we categorized the TASO-CDDP model as being highly task-shifted because the services are delivered at community outreach settings and the TASO-C model to be minimally task-shifted because the ART services are delivered at health facility clinics.

The operational service delivery unit in TASO is called a center. TASO has 11 centers that are distributed across the 10 regions of the country. Each TASO center serves three to five districts. For this reason, the selection unit for TASO was a center rather than a district. Out of the 11 TASO centers, five were purposively selected to ensure fair regional representation: Entebbe (Central 1 region), Jinja (East-central), Gulu (Mid-north), Masindi (Mid-west), and Rukungiri (South-west). The central, minimally taskshifted clinic of each of these centers was included as a study site. In addition, for each TASO Center two community drug distribution points (CDDPs), the highly-task-shifted clinics, were purposively selected to ensure fairness in geographical and low- vs high- volume distribution. One high volume and one low volume CDDP site was chosen from each center, resulting in the following 10 CDDPs: Bayita, BuhimbaKaruma, Buyanja, Dwoli-Biiso, Kanyinya, Kanyogoga, Kiyindi, Layibi, Masajja-Kawoto, and Red Cross.

\section{DATA COLLECTION METHODS AND ANALYSIS}

\section{Facility audits}

Facility audits were conducted in all 28 participating clinics by senior nurses who had received a fiveday training on data collection for this study. They visited the selected health facilities and observed the delivery of ART for one full day, from the time the first patient was served up to when the last patient was served. The facility audits were conducted at four Kitovu Mobile outreach clinics, six UC-MOH clinics, three UC-S clinics, 10 TASO-CDDP clinics, and five TASO-C clinics. The observers completed a quantitative facility audit tool and the data were entered in Epi Data and analyzed in Microsoft Excel to generate simple frequencies for the following key study outcomes: opening and closing hours, number and types of HWs present that day at the clinic, number of patients seen at the clinic, and the ART tasks performed by each cadre of HWs. The facility audits also provided information about delegation of tasks by documenting the cadres of health workers performing various tasks in ART care on the day of the audit. In the analysis, the cadres documented by the facility audits as performing certain ART tasks were compared to the cadres that, according to the World Health Organization (WHO 2008), were conventionally supposed to perform those tasks.

\section{Client-provider interaction (CPI) observations}

At each clinic, at least 50 CPIs were observed, except at UC-S where only 45 sessions were available for observation. Fifty patients at each clinic was deemed to be a feasible number that could be observed in one or two days. This sample size is sufficient for this descriptive study.

Prior to observing a service delivery session the nurse-observers sought consent from the HW to observe a session that the HW was about to conduct with a patient. After obtaining the HW's signed consent, the observer was introduced to the patient by the HW and the observer sought the patient's signed consent to observe the session. The observers were trained to remain silent and unobtrusive during the session. They used a checklist of key ART care tasks which they checked using a three point scale of "Yes," "Somewhat," 
and "Not at all" to indicate whether, and how well, each task was performed by the HW during the session.

The performances of HWs of the following key ART tasks were observed:

- Assessment of the patient (weighing, asking about complaints, exploring issues);

- Adherence counseling (asking about: missed dose, challenges in adherence, and family support; discussing adherence on ARVs received that day);

- Side effects counseling (asking about side effects; discussing signs and symptoms of side effects and what should be done in case of side effects); and

- Addressing complaints (providing solutions or referrals for each complaint raised by a patient).

These data were analyzed by creating an index of performance for each task observed. Each task for which the observer's response was "Yes" was scored two while the "Somewhat" response was scored one and the "Not at all" response was scored zero. A sum of these values was then obtained for each task, which became the numerator for calculating the percentage performance score for that task. The denominator was obtained by multiplying the number of sessions observed by a factor of two which represents the highest possible performance score ("Yes").

\section{Time-motion study}

At each of the 28 selected clinics, a sample size of 50 patients was conveniently recruited for the timemotion component. Patients were followed by research assistants (RA) with a social science background who had received a five-day training on data collection for this study. Each patient arriving at the health facility was approached by a RA who sought their consent to be followed by the RA at all the stations of the clinic as they received services. Using a time-motion study tool the RA recorded the time the patient started receiving the service at each station, the cadre of the provider who served the patient at that station, and the time the patient left that station. Simple statistics were generated describing patient's time spent at each station, time spent waiting between stations, and overall time spent at the health facility.

\section{Client exit interviews}

For the exit interviews component of the study, a sample size of 78 was calculated as sufficient for detecting a difference of 15 percent in client satisfaction between two types of task-shifting models (95 percent $\mathrm{CI}$ and 80 percent power). A sample size of 80 patients was therefore selected in each clinic. The interviews were conducted by RAs with a social science background who had received a five-day training on data collection for this study. Descriptive data analysis was used to determine patient knowledge about ARVs, patient recall of ART care services received during the clinic visit, and satisfaction with services received.

Regarding $A R V$ knowledge, the respondents were asked about the following aspects: benefits of ARVs, side effects of ARVs, adherence requirements of ARVs, and what happens if one is not adhering to ARVs. The proportion of patients who correctly answered each of these questions was calculated separately, after which a simple average of these percentages was computed to represent the ARV knowledge level of the respondents. 
On recall of services received, the respondents were asked if they recalled receiving the following discussion or service from the provider ${ }^{3}$ in the clinic that day: talked with a HW about ARVs, remaining pills counted, asked by HW about missing an ARV dose, and asked by HW whether taking other medicines. The proportion recalling each of these services was calculated separately for each service, after which a simple average was computed to represent recall of services received.

With regard to client satisfaction, the exit interview asked the following questions: whether the patient felt that the HW listened to them and gave them a chance to ask questions, whether the patient was satisfied with the time spent at clinic, whether they felt they had enough privacy during the consultation, whether the discussion was helpful, and whether they would come back to that clinic to seek services in the future. The proportion answering positively on each of these questions was calculated separately for each question, after which a simple average was computed to represent satisfaction.

\section{Interviews with health workers}

HWs working in the study facilities were interviewed by RAs with a social science background who had received a five-day training for data collection. The RAs interviewed as many of the HWs present at the clinic on the day the study team visited the clinic as possible; in some cases they could not interview HWs who were too occupied with care activities. The health worker interviews were administered to a total of $268 \mathrm{HW}$ s found working at these clinics on the day of the study, 150 (56 percent) of whom were professional health workers while the rest were lay health workers. The breakdown per task-shifting model is $40 \mathrm{HWs}$ from Kitovu Mobile; 60 from UC-MOH, 28 from UC-S, 72 from TASO-CDDP, and 68 from TASO-C. The data were entered into Epi data and were analyzed in Excel for simple frequencies on the following key outcomes: ART care tasks delegated out from the health worker and tasks delegated to the health worker, as well as ART training received.

\section{Review of program reports}

The latest available annual reports for each ASO were reviewed to extract information about the history of the organization, the number of districts and health facilities served by the ASO, and the number of HIV patients enrolled in pre-ART and ART care. Information about the task-shifting approach of the ASO was also obtained.

\section{Review of service statistics}

Data from clinical records of all patients aged 16 years and older who attended ART services in all clinics run by the three organizations between July 2010 and December 2012 were collected. The following information was obtained:

- Date of initiation of ART

- Personal information: age, sex, clinic ID number

- Dates of return visits

- Types and quantity of all ARV medications delivered to the patient

${ }^{3}$ Health workers were not informed that their patients would be asked whether they received these discussions or services 
To collect these data, trained data extractors visited the central offices of TASO, UC, and Kitovu Mobile to review patient visit records from the organization's electronic database. The data collectors constructed patient histories by linking individual visit records over time, using unique patient identification numbers. Only patient records that included the following were included in the analysis.

1. A valid date and visit location.

2. Whether the patient was to receive additional ART medication.

3. The types of ART dispensed, or documentation of the patient's assigned ART regimen.

4. The number of pills of each drug dispensed.

The completed service statistics data were imported to the STATA 11 statistical package for final cleaning and analysis. Using the available data, a number of variables were constructed to assess retention in care:

1. Inter-visit period: The number of days between two successive ART refill visits.

2. Drug adherence: The number of days the medication dispensed to a patient at each refill visit was expected to last. For example, if a patient was prescribed an ARV drug regimen where one pill is taken twice daily, and dispensing records indicate that 60 tablets were dispensed to the patient on his/her last refill visit, then that patient had 30 days of drug coverage before the next refill visit. Patients who do not attend a refill visit before their dispensed drugs are exhausted are considered non-adherent for the additional days.

3. Default: Patients who had exhausted and remained non-adherent for more than 45 consecutive days before receiving a documented refill were considered to be in default on planned treatment. The buffer period of 45 days was selected for consistency with other ART programs.

4. Resuming care: Any patient who, after defaulting had returned for clinical services before December 2012.

5. Treatment gaps: the number of times that a patient defaulted and subsequently resumed care between July 1, 2010 and December 31, 2012.

6. Loss to follow-up: Any patient was considered lost to follow-up if s/he defaulted on clinical services, failed to resume care prior to December 31, 2012, and had no ultimate outcome recorded in the clinical registry. The buffer period of 45 days was selected for consistency with other ART programs. For this study, any patient who had entered default on or before November 18, 2012, did not complete another documented visit by December 31, 2012, and had no documented reason for failing to attend a refill visit was deemed lost to follow-up.

7. Total time in care: The number of days elapsed between a patient's ART initiation date and their last documented visit prior to December 31, 2012.

We assessed the two key patient-level outcomes based on the above variables:

1. Retention in care: Patients were considered retained in care if they were not lost to follow-up as of December 31, 2012. 
2. > 95 percent drug adherence: The proportion of patients, for whom at least 95 percent of their total time in care was adequately covered by the ARV medication dispensed at their documented refill visits. This indicator was used as a surrogate measure for adherence.

For TASO facilities only, association between the two outcomes and two task-shifted models (TASO-C and TASO-CDDP) were also assessed using descriptive and bivariate data analysis. These associations were not examined for Kitovu Mobile and UC sites due to data limitations, as discussed in the study limitations section.

\section{Collection of costing data}

Costing analysis was undertaken from the provider's perspective. Analysis only included costs that were directly incurred by the provider, and excluded costs covered by clients. Ancillary and opportunity costs incurred by patients such as transportation and time were not collected.

The ART drug costs, number of clients, number of visits, operational costs, and other costs related to service delivery were collected retrospectively using routine monitoring and evaluation (M\&E) data from the three participating organizations, including accounting records, client visit logs, ART distribution logs, equipment inventories, and routine reports. Salaries of staff providing ART services were collected based on payroll records, including both salary and benefits. Time spent on ART service delivery was determined based on interviews with staff and their managers. The time spent on ART was then calculated as a ratio of the total number of hours spent on ART divided by the total working hours per day (eight hours per day). The percentage of office rent and operational costs attributed to ART was calculated based on the percentage of the ART program budget in relation to the total budget for the organization.

Four TASO centers out of 11 total, and eight out of 29 UC clinics were randomly selected for the cost study. Because Kitovu Mobile is much smaller in size and operates in only one region, all 111 Kitovu Mobile outreach mobile units were selected. Data were collected for one calendar year. We chose to collect the 2012 data to get the most recent data that would also be considered relevant and useful. Monthly 2012 data from sampled facilities were collected then aggregated for the whole year.

Apportionment of shared costs: Apportioning costs for staff not full time on ART service delivery was estimated based on percentage of time spent on ART service delivery. For TASO, which has a regional structure, personnel costs at the national level were equally shared among the four TASO centers in the four regions. At Uganda Cares and Kitovu Mobile, headquarter operational costs attributed to ART were equally distributed among all the service delivery points of the organization. 


\section{RESULTS}

\section{HIVTREATMENT TASK-SHIFTING APPROACHES OF PARTICIPANT ORGANIZATIONS}

This section presents the description of the task-shifting approaches of the three ASOs studied, including what tasks are delegated, which cadre is involved in task-shifting, at what locations task-shifting is taking place, and how the task-shifting approaches were developed.

\section{Kitovu Mobile}

Kitovu Mobile has been providing ART care since 2006 through 111 outreach units in the hard-to=reach communities. Since Kitovu Mobile does not have a central clinic, its HIV care services are entirely delivered at community locations such as a church, the verandah of a home, or under a tree. According to their service statistics, by June 2014 Kitovu Mobile had 1,833 current patients, of which 1,439 were on ART; and had enrolled 110 new ART patients from January-June 2014.

How the ART task-shifting approach was developed

A review of program reports showed that the following measures were undertaken to integrate ART care into the community outreach model of Kitovu Mobile:

a. Training of expert patients in ART care: The term "expert patients" refers to ART patients who have received ART care for some time and have exhibited a high degree of understanding of ARV side effects and adherence issues. They are then selected and given a short training in ART adherence counseling and community education about ART, and become employed by Kitovu Mobile to educate and counsel other patients about ART. At the time of our study Kitovu Mobile had trained 177 expert patients who functioned as treatment supporters for fellow patients and assisted nurses in the conduct of the clinic.

b. Establishment of community outreach clinic locations: Kitovu Mobile was established as a home-based care program that provided HIV care and other palliative care services to PLHIV by visiting each patient at home. When the number of patients increased, a decision was made to ask the patients to gather in one community outreach site where a visiting Kitovu Mobile team would meet them on the appointed day. This is the model that is being used currently to provide pre-ART and ART care.

c. Process of the community outreach settings: At each site the clinic takes place once a month and the day of the clinic is well known to the community via word of mouth and messages from village health team (VHT) members. In addition, at each clinic day existing patients get an appointment for their next clinic visit. Determining patient eligibility for ARVs, including collecting blood for CD4 counts, is done by nurses who then develop a list of patients needing initiation on ARVs and make appointments for the ART initiation team to meet the patients at their respective community outreach site. The ART initiation team is led by a clinical officer. Once patients are initiated on $\mathrm{ARV}$ s and have been well stabilized, they receive follow up from a nurse-led team at the community 
outreach site once a month to get their ARV refills and receive other HIV care. The nurse-led team includes expert patients who provide education to waiting patients about the procedures of ART initiation as well as the importance of adherence.

d. Supervision: When the clinical officer visits the community outreach settings to initiate new patients on ART s/he also provides supervision to the nurses and expert patients and is able to examine and treat any of the ART refill patients who the nurse identifies with additional clinical problems requiring further care.

The data in Table 3 are from 37 HW interviews from Kitovu Mobile comprising 11 PHWs (1 clinical officer, 4 nurses, 4 counselors, and 2 lab workers) and 26 LHWs (2 community health workers and 24 expert clients). It shows that in Kitovu Mobile the majority (9 out of 11) of PHWs and all 26 LHWs had undergone a specific training on ART care. Regarding specific skills, however, a low proportion of PHWs reported training in any of the four key skills: prescribing (4/11), dispensing (3/11), counseling (5/11), and health talk (2/11). In contrast, the majority of LHWs reported having been trained in the two skills of counseling, 88 percent (23/26), and health talk, 73 percent (19/26) — the two roles most delegated to LHWs. It would have been interesting to examine proportions trained by each specific cadre (e.g., nurses) but the sample sizes of each cadre were too small for comparison.

Table 3 Kitovu Mobile providers reporting ART-care training

\begin{tabular}{|lcc|}
\hline \multicolumn{2}{|l|}{ Professional HWs (clinical officers, nurses, counselors, lab workers) } & $\mathbf{n}=\mathbf{~ I ~}$ \\
\hline \multicolumn{2}{|l}{ Has received specific ART-care training } & 9 \\
\hline Topic of ART-care training: & Prescribing & 4 \\
& Dispensing & 3 \\
& Counseling & 5 \\
& Health talk & 2 \\
\hline LHWs (Expert clients, VHT) & & $\mathbf{n}=26$ \\
& Has received specific ART-care training (Prop) \\
\hline Topic of ART-care training & Prescribing & $26(1.0)$ \\
& Dispensing & $6(0.2)$ \\
& Counseling & $9(0.3)$ \\
& Health talk & $23(0.9)$ \\
\hline
\end{tabular}

Data source: HW interviews; more than one response possible.

The data from the facility audit tool showed that among the four Kitovu Mobile clinics in the study the average number of patients on the day of observation was 50 per clinic; it was observed that there were an average of 3.25 nurses per clinic and 3.5 expert clients per clinic serving that day. Thus, the workload per nurse was similar to the work load per expert client-14 to 15 patients per provider.

Table 4 shows how clinical tasks were delegated at Kitovu Mobile. It is shown that clinical review, counseling, and dispensing that are conventionally supposed to be performed by clinical officers, 
counselors, and pharmacy workers, respectively, were documented as being performed by nurses, and preclinical tasks were performed by LHWs, implying that at Kitovu Mobile, which only has a highly taskshifted model, both the pre-clinical and clinical ART care tasks were delegated.

Table 4 Tasks delegated at Kitovu Mobile

\begin{tabular}{|c|c|c|c|c|c|c|}
\hline & \multicolumn{6}{|c|}{ Performer of task } \\
\hline & 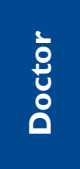 & 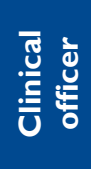 & 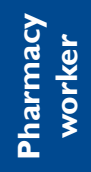 & $\begin{array}{l}\frac{2}{0} \\
\frac{0}{0} \\
0 \\
0\end{array}$ & $\frac{\emptyset}{2}$ & 3 \\
\hline Pre-clinical (registration, triage, health talk, and vital signs) & $\mathrm{N} / \mathrm{A}$ & & & & 0 & $\mathbf{0}$ \\
\hline Clinical review & $\mathrm{N} / \mathrm{A}$ & م & & & $\mathbf{0}$ & \\
\hline Counseling & N/A & & & 0 & $\mathbf{0}$ & $\mathbf{0}$ \\
\hline Dispensing & $\mathrm{N} / \mathrm{A}$ & & 0 & & $\mathbf{0}$ & \\
\hline
\end{tabular}

- Conventional performer of task; $\mathbf{O}$ - Observed performer of task Data sources: facility audits $(n=4)$

\section{Uganda Cares (UC)}

UC is operated by the AIDS Healthcare Foundation (AHF) ${ }^{4}$ in partnership with the Ministry of Health $(\mathrm{MOH})$ and has provided HIV care in Uganda since 2002. According to their service statistics, UC had 50,039 current HIV patients; including 16,000 new patients in 2013.

\section{How the ART task-shifting approach was developed}

A review of program reports showed that UC, which was initiated in the ART era, undertook the following measures to address the overwhelming workload of ART care on the few professional HWs available:

a. Training of HIV medics: UC has a task-shifting approach that uses a cadre of trained lay health workers known as "HIV medics" to assist in the provision of ART care. The HIV medic model of UC started in 2004. It entails recruitment of lay people with a basic education of at least four years of secondary school who are then given a 12 week didactic and practical training course in basic HIV care, triage, and intensive adherence support in the provision of ART and ART monitoring.

b. Training of expert clients: In addition to HIV medics UC trains expert clients, who are selected from among their HIV patients, to support the care of other patients.

c. Clinic process: During the ART clinic HIV medics assist doctors, clinical officers, and nurses by providing the following services: basic triage, intensive adherence support, health education to waiting patients, ARV drug refills for stable patients, phlebotomy, pharmacy inventory, and logistics management. Expert clients also assist in patient triage including weighing, taking body temperature, and measuring height as well as the mid upper arm circumference for assessing nutrition status. They

${ }^{4}$ http://www.aidshealth.org/\#/ 
also assist with records management which includes chart retrieval and returns, filling of records such as registers, inserting test results into patient files, and moving charts to and from clinician rooms. In addition they help in transporting blood samples from lower health units to reference laboratory. They also provide patient education and counseling and help track patients in care, telephoning patients who have missed appointments as well as those who need review before the scheduled visit date. They further assist with the cleaning and dusting of the clinic area.

d. Training and supervision: UC has a team comprising a medical doctor and nurses that conduct training and visits to clinics in various parts of the country to provide supportive supervision to staff. The day-to-day supervision of both HIV medics and expert clients is entrusted to nurses, since this is the department they work with very closely. Additional support, mentorship, and training are availed by the program nurse manager as well as training manager. The health workers also receive ongoing training through clinical team meetings as well as site level continuing medical education (CME) sessions.

The data in Table 5 are from the interviews of 92 HWs from UC comprising 50 PHWs (1 doctor, 9 clinical officers, 18 nurses, 10 counselors, 2 pharmacy workers and 10 lab workers); and 42 LHWs (22 expert clients, $12 \mathrm{HIV}$ medics, and 8 counseling aides). It shows that when we combine UC-MOH with UC-S, 100 percent (50/50) of PHWs and a majority of LHWs, 93 percent (39/42), reported having undergone a specific training on ART care. Regarding specific skills, half or more of PHWs reported training in any of the four key skills: prescribing, 50 percent (25/50), dispensing, 52 percent $(26 / 50)$, counseling, 62 percent (31/50), and health talk, 54 percent (27/50). A slightly higher proportion of LHWs reported having been trained in the two skills of counseling, 71 percent (30/42) and health talk about ART, 67 percent (28/42), which are the two roles most delegated to LHWs. It would have been interesting to examine proportions trained by each specific cadre (e.g., clinical officers) but the sample sizes of some cadres were too small for comparison. However, among PHWs nurses had a substantial sample size $(\mathrm{n}=18)$ and among these about three quarters were trained in prescribing $(13 / 18)$ and about two thirds in dispensing, counseling, and health talk (11/18) (data not shown). Thus these small numbers suggest that a higher proportion of nurses (2/3) than other PHWs at UC (1/2) were trained in key skills.

The facility audits documented a wider variety of health workers at UC sites compared to Kitovu Mobile sites, including doctors, clinical officers, nurses, counselors, pharmacy workers, and laboratory workers; and lay health workers (expert clients, as defined above, and HIV medics). On the day of audit the UC$\mathrm{MOH}$ clinics had a lower number of patients, averaging 44 per clinic, compared to 94 patients per UC-S clinic. The audits also showed that the work load per professional health worker was similar at both UCMOH and UC-S, 11 patients that day. The work load per LHW was however lower at UC-MOH than UC-S clinics, nine patients compared to 15 patients. 
Table 5 UC providers reporting ART-care training

\begin{tabular}{|c|c|c|c|}
\hline & & UC-MOH & UC-S \\
\hline $\begin{array}{l}\text { Professional HWs (doctors, } \\
\text { workers, lab workers) }\end{array}$ & officers, nurses, counselors, pharmacy & $\begin{array}{c}n=34 \\
\text { n (Prop) }\end{array}$ & $n=16$ \\
\hline Has received specific ART-c & ing & $34(1.0)$ & 16 \\
\hline Topic of ART-care training: & Prescribing & $17(0.5)$ & 8 \\
\hline & Dispensing & $18(0.5)$ & 8 \\
\hline & Counseling & $22(0.6)$ & 9 \\
\hline & Health talk & $21(0.6)$ & 6 \\
\hline LHWs (Expert clients, HIV & counseling aides) & $\begin{array}{c}n=30 \\
n \text { (Prop) }\end{array}$ & $n=12$ \\
\hline Has received specific ART-c & ing & $29(1.0)$ & 10 \\
\hline Topic of ART-care training: & Prescribing & $8(0.3)$ & 6 \\
\hline & Dispensing & $9(0.3)$ & 6 \\
\hline & Counseling & $23(0.8)$ & 7 \\
\hline & Health talk & $20(0.7)$ & 8 \\
\hline
\end{tabular}

Data source: HW interviews; more than one response possible.

As Table 6 shows, in UC clinics there was a variation in the model of delegating clinical tasks. At UC$\mathrm{MOH}$ the facility audits documented that clinical review, which is conventionally the role of doctors and clinical officers, was being performed by only clinical officers. Counseling and dispensing were conducted by nurses, implying that in this model these tasks, which conventionally are the roles of counselors and pharmacy workers, respectively, were delegated ${ }^{5}$ to nurses. The delegation of tasks at UC-S was similar to UC-MOH except for clinical review, which during the facility audits was observed to be performed by both doctors and clinical officers, suggesting that this task was not delegated. However, UC programs reported that for some stable ART patients coming for ARV refills, the task of clinical review is sometimes delegated to nurses and HIV medics.

${ }^{5}$ Delegation of tasks does not always imply that the HW being delegated to is lower in rank, but it could mean that the HW being delegated to is not conventionally the one designated to perform that role. For example delegation of counseling from a counselor to a nurse does not imply that nurses are a lower rank to counselors-they are in different professions each with its own hierarchical structure so they cannot be ranked against each other. 
Table 6 Tasks delegated at UC

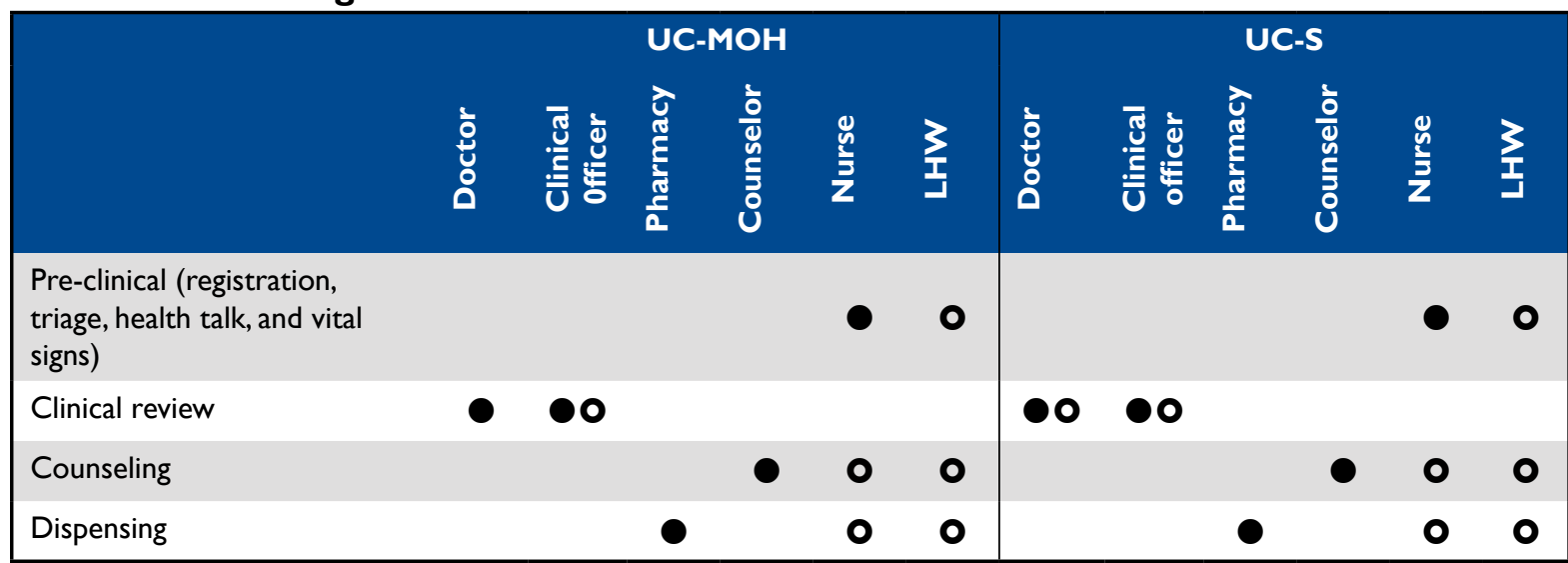

- Conventional performer of task; $\mathbf{O}$ - Observed performer of task

Data sources: Facility audits $(\mathrm{n}=9)$

\section{The AIDS Support Organization (TASO)}

TASO $^{6}$ is a non-governmental organization that was among the first to provide HIV care and support in Uganda and sub-Saharan Africa in 1987 and has provided ART to its patients in Uganda since 2004. Their service statistics showed that as of June 2014 TASO had 91,218 current patients of whom 68,584 were on ART, including 13,394 who were new ART patients in 2013.

TASO uses a hierarchical structure of one national headquarter office working through four subnational offices to support the 11 service delivery centers. ART patients attending TASO-CDDP receive monitoring by a visiting team of clinicians and counselors every six months.

\section{How the ART task-shifting approach was developed}

A review of program reports showed that to institute the CDDPs, TASO undertook the following measures:

a. Training of expert clients in ART care: Each TASO center has community ART support agents (CASAs) who are expert clients on ART and who were trained to mobilize other patients to attend the drug distribution point and to provide counseling and monitoring of adherence to a maximum of 10 patients within their locality. CASAs are ART patients who can read and write, and who are selected by TASO with the consent of other patients residing in a specific community. These patients are trained on basic information about ART and HIV/AIDS in general and given skills to conduct a number of activities including refilling of ARVs, giving out CD4 results, counseling, and checking hemoglobin levels. They are also equipped with bicycles, gumboots, and TASO T-shirts, bags, books, and pens to facilitate their work.

${ }^{6} \mathrm{http}: / /$ www.tasouganda.org/ 
b. Establishment of community outreach locations: When TASO decided to start CDDPs, the expert clients were asked to consult with patients in their group to identify a venue in the community where they could congregate to receive ARVs from a TASO team visiting from the central clinic. After the sites were selected, about 30 patients were grouped in one community site which became a CDDP. Each TASO center has about 30 CDDPs and the locations include schools, community halls, places of worship, and sometimes just under a large tree.

c. Training counselors in ARV refill: At each TASO center there is a cadre of counselors (counseling diploma holders) whose traditional role had been to provide counseling, including pre- and post-test counseling and adherence counseling. When the CDDP model was introduced the counselors were trained in dispensing ARV refills. They were also trained in conducting hemoglobin tests using test strip technology to identify anemia as a sign of toxicity. Additionally, they were trained to conduct pregnancy tests to identify early pregnancy since, at that time, the ARV Efavirenz was not considered safe in early pregnancy (WHO 2012).

d. Process of the CDDP clinic: ART patients who had stabilized on medication were selected and downreferred to a TASO-CDDP of their choice to receive ARV refills on an ongoing basis. Each CDDP was allocated one day every other month when the counselor would visit the outreach clinic to deliver the ARV refills. In the morning of that date the counselor works with the pharmacy staff at the central clinic to pack ARV refills for each patient for the given CDDP. The counselor then transports these ARV packets in a car to the community outreach setting. At the outreach clinic the process starts with a health education talk for all patients followed by a one-on-one consultation during which the counselor asks about the patients' well-being, conducts the hemoglobin test and pregnancy test (for females), dispenses the ARVs, and provides adherence counseling. The counselor is assisted by expert patients in providing this care. CDDP patients with medical and psychosocial issues are upreferred to TASO-C for the review after which they are down-referred to the TASO-CDDP to resume ongoing ARV refill.

e. Supervision: Each CDDP care team (the counselor and the expert clients) is supervised by a doctor or clinical officer and a nurse to ensure that the patient care is in line with TASO's quality of care. The supervising team moves among the CDDPs every six months observing the care provided, including reviewing some patients and providing support as needed.

The data in Table 7 are from the HW interviews of 126 HWs from TASO comprising 89 PHWs (4 doctors, 13 clinical officers, 12 nurses, 41 counselors, 7 pharmacy workers, and 12 lab workers); and 37 LHWs (30 expert clients, 2 community health workers, and 5 counseling aides). It showed that in TASOCDDP combined with TASCO-C the majority of PHWs, 83 percent (74/89), and of LHWs, 84 percent (31/37), reported having undergone training on ART care. Regarding specific skills: about half of PHWs reported training in any of the four key skills: prescribing, 39 percent (35/89); dispensing, 46 percent (41/89); counseling, 56 percent (50/89); and health talk, 40 percent (36/89). Among the LHWs there was a wide variation in the proportion reporting having been trained in the two skills most delegated to LHWs_-49 percent (18/37) trained in counseling compared to 68 percent (25/37) trained in health talk. It would have been interesting to examine proportions trained by each specific cadre (e.g., nurses) but the sample sizes of some cadres were too small for comparison. However, among PHWs, counselors had a substantial sample size $(n=41)$; among these 29 percent $(12 / 41)$ were trained in prescribing, 51 percent 
(21/41) in dispensing, 85 percent (35/41) in ARV counseling, and 49 percent (20/41) in health talk. Of particular interest are the counselors in the TASO-CDDP model who are responsible for dispensing ARV refills, of whom only 60 percent (12/20) reported training in dispensing of ARVs (data not shown).

\section{Table 7 TASO providers reporting ART-care training}

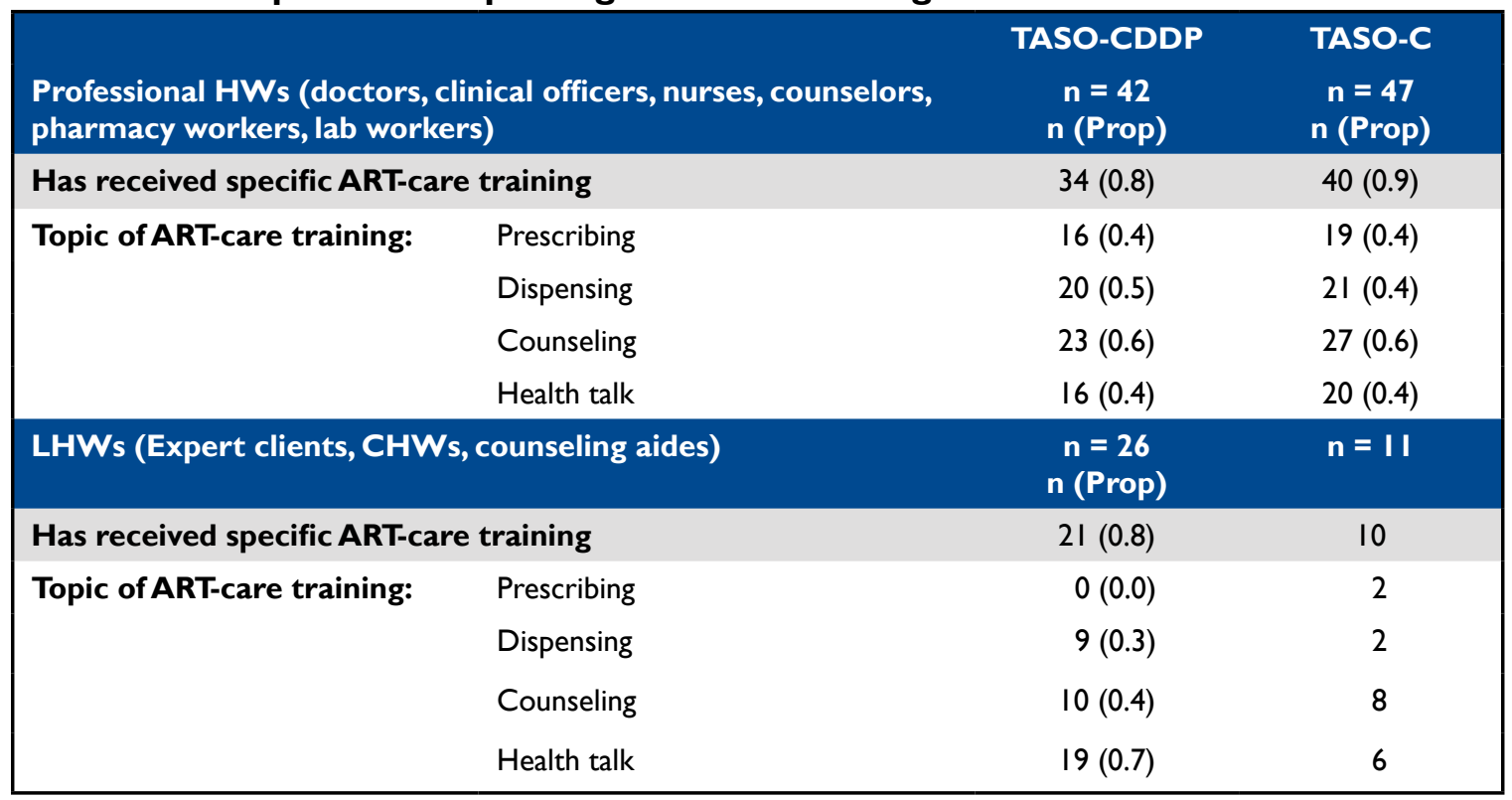

Data source: HW interviews; more than one response possible.

The facility audit data showed that the 10 CDDPs observed served an average of 56 patients per clinic, compared to the central clinics that served an average of 119 patients per clinic on the day of observation. There were 38 professional health workers and 10 LHWs documented at the 10 CDDPs altogether, while at the central clinics there were 102 professional health workers and 19 LHWs. Thus the daily work load per professional health workers was higher at the CDDP clinics (15 patients) compared to the central clinic (6 patients). The workloads for lay HWs at both the CDDP and the central clinics were quite high, 56 and 32 respectively, suggesting that each lay HW spent little time with each patient, probably carrying out only simple tasks like triage.

Table 8 shows that at the TASO-CDDP sites, clinical review-which is conventionally supposed to be performed by doctors and clinical officers - was documented in the facility audits as being performed by nurses. Counseling — conventionally supposed to be performed by counselors-was being performed by LHWs. Dispensing of ARVs—a conventional role of pharmacy workers—was being carried out by counselors assisted by LHWs. These data imply that all ART care tasks at TASO-CDDP, a highly taskshifted model, were delegated from the providers who were conventionally supposed to perform them to other health workers.

It is further shown that at TASO-C, clinical review was performed by both doctors and clinical officers who are conventionally supposed to perform this task. Similarly, dispensing of ARVs was being performed by pharmacy workers, the cadre that is supposed to do this job. Counseling was being performed by 
counselors and LHWs. Thus, at TASO-C, a minimally task-shifted model, there was no delegation of clinical review and dispensing while there was some delegation of counseling to LHWs.

Table 8 Tasks delegated at TASO

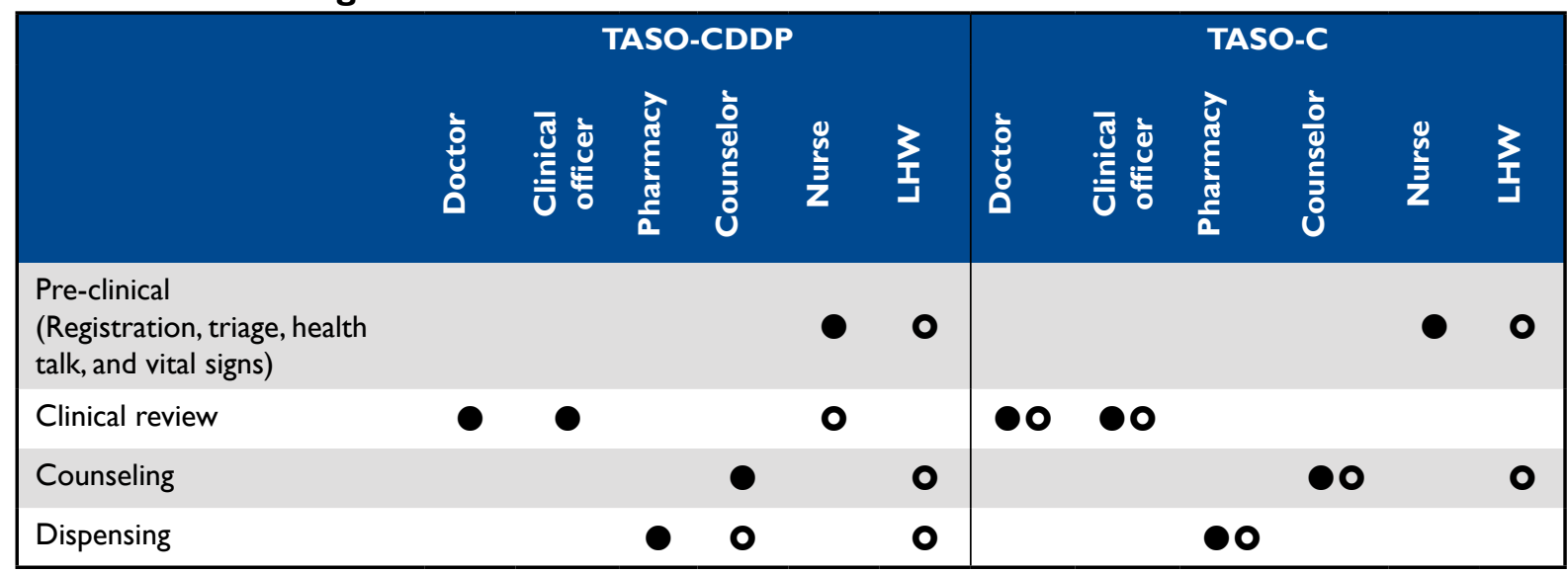

- Conventional performer of task; $\mathbf{O}$ - Observed performer of task

\section{Overview of delegation of tasks}

Figure 3 examines delegation of tasks for the three ASOs combined. As expected, it shows that tasks tended to be delegated from the health workers at the apex of the medical order-doctors and clinical officers - rather than to them. For the mid-level health workers-nurses and counselors - there was similar likelihood of tasks being delegated from them and to them. As for providers toward the bottom of the rank-expert clients and HIV medics - there was an inclination to delegate tasks to them, rather than from them.

Figure 3 Overview of delegation of tasks

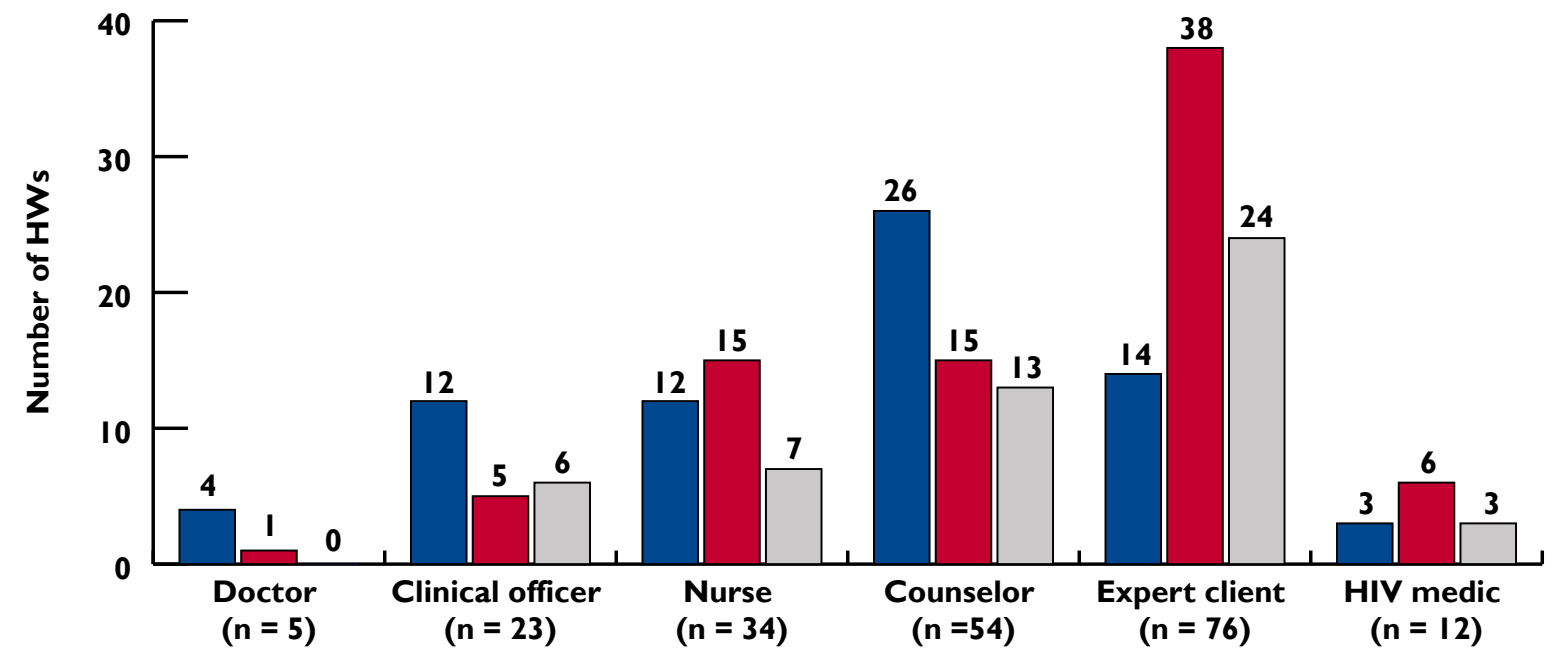

$\square$ Delegated from $\square$ Delegated to $\square$ Neither or missing

Data source: Health worker interviews $(n=268)$ 
However, it is notable that doctors, who are at the apex, also reported that some tasks were delegated to them. Similarly, expert clients and HIV medics, who are at the bottom ranks, also reported that some tasks were delegated from them. Inquiry from program managers revealed that doctors could get ART care tasks delegated to them by other doctors who are more senior. The program managers also reported that HIV medics and expert clients could delegate some simple tasks, such as triage, to ordinary ART patients to assist on a busy day.

The pattern of delegation emerging from HW interviews demonstrated in Figure 1 seems to confirm the pattern that emerged from facility audits, shown in Tables 4, 6 and 8. Both data sources show that the two mid-level cadres (counselors and nurses) were as likely to report tasks being delegated from them as tasks being delegated to them. For example, the facility audits showed that the role of nurses to perform pre-clinical care was delegated to LHWs in all the models, while nurses were having the roles of clinical assessment, counseling, and dispensing being delegated to them in some models. Similarly, at TASOCDDP, facility audits showed that counseling was delegated from counselors to LHWs while counselors got the role of dispensing delegated to them.

Another way to look at the pattern of delegation is by type of task-shifting model (Figure 4). The data show that in the community-based models (Kitovu Mobile and TASO-CDDP) the HWs were more likely to report being delegated to ( 60 percent and 51 percent respectively) than to report being delegated from (38 percent and 32 percent respectively). In conformity, at UC-S, a facility-based model, the HWs were more likely to report being delegated from (29 percent) than being delegated to (18 percent). However, at TASO-C and UC-MOH no trend emerges. Thus, excluding TASO-C and UC-MOH, the data suggest that in the community-based settings the HWs were more likely to be delegated to compared with the facility-based clinic.

\section{Figure 4 Pattern of delegation by model of care (HW interviews)}

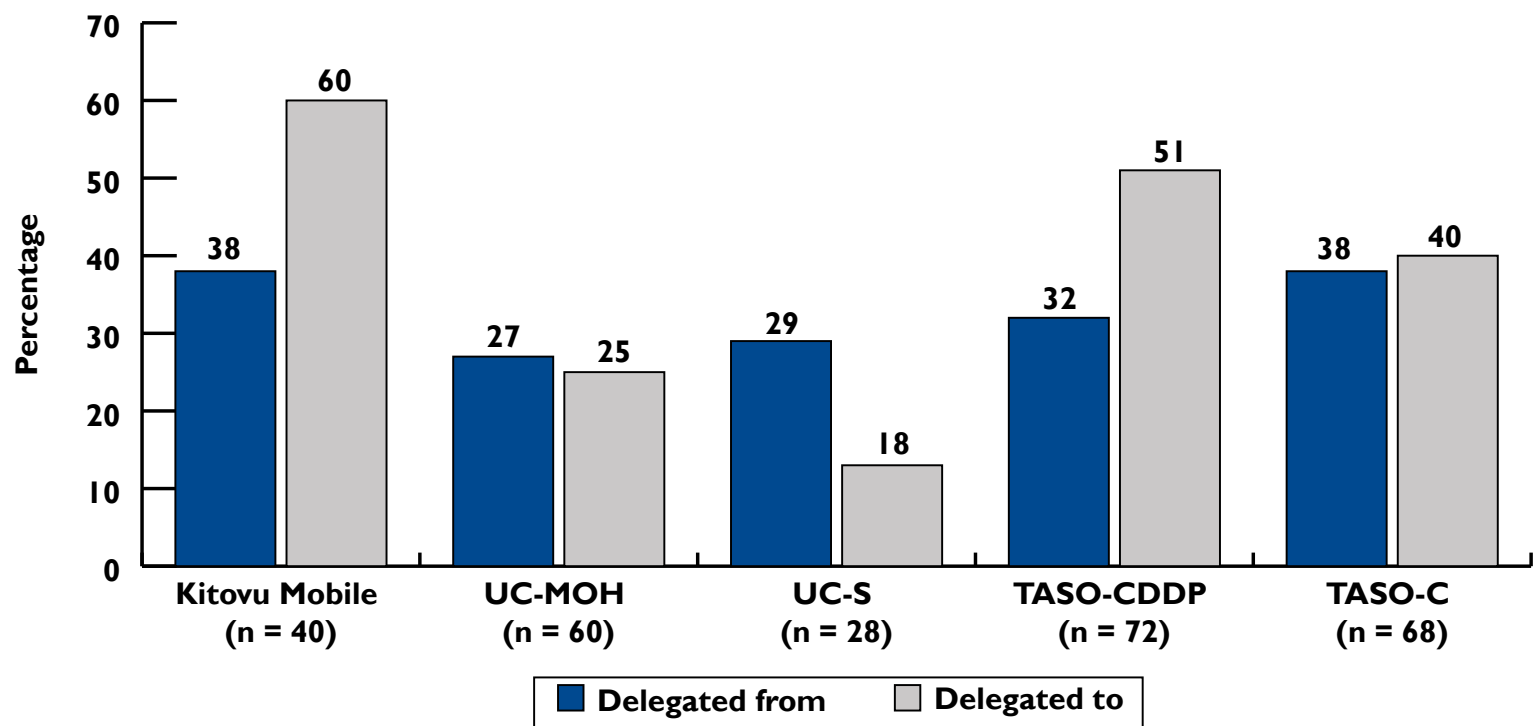




\section{PATIENT-LEVEL OUTCOMES}

This section focuses on the implications of task-shifting on patient level outcomes.

\section{Client perceptions}

Table 9 compares data from the community-based settings (Kitovu Mobile and TASO-CDDP) to the facility-based clinics (UC-S, UC-MOH, and TASO-C) with regard to three patient outcomes: ARV knowledge, recall of services received, and client satisfaction. In both the community-based and the facility-based clinics, only about one-third of the patients gave correct responses to the ARV knowledge questions. The data show that the proportion recalling these key services was marginally higher in the facility-based (66 percent) compared to the community-based clinics (59 percent) $(\mathrm{p}<0.05)$.

Table 9 Self-reported patient-level outcomes in all models (Client exit survey)

\begin{tabular}{|c|c|c|c|}
\hline Respondents & $\begin{array}{l}\text { Community-based } \\
\begin{array}{c}n=1,166 \\
\text { Prop }\end{array}\end{array}$ & $\begin{array}{c}\text { Facility-based } \\
\begin{array}{c}n=1,108 \\
\text { Prop }\end{array}\end{array}$ & p-value \\
\hline ARV knowledge index & 0.32 & 0.35 & $>0.05$ \\
\hline Recall of key ARV services index & 0.59 & 0.66 & $<0.05$ \\
\hline Satisfaction index & 0.90 & 0.93 & $<0.05$ \\
\hline
\end{tabular}

The data show that client satisfaction was quite high overall and marginally higher at the facility-based (93 percent) compared with the community-based clinics $(90$ percent $)(p<0.05)$.

In summary, these findings suggest that client knowledge about ARVs is low in all the clinics. However, patient recall of receiving key elements of discussion about ARVs with the provider in the clinic that day and client satisfaction were marginally higher at the facility-based compared to the communitybased settings. This difference in recall, while statistically significant, was programmatically marginal at 7 percentage points, and it is important to note that client satisfaction was quite high in both communitybased and facility-based clinics.

\section{Retrospective analysis of routine service data}

Table 10 shows the patient histories that were collected from the three ASOs: 1,944 patient histories were collected from five TASO facilities, 198 patient histories from two Uganda Cares sites, and 81 patient histories were obtained from Kitovu Mobile. Overall, TASO patients were more frequently female; Uganda Cares patients were younger and were more evenly distributed by gender than TASO patients. Patients of Kitovu Mobile were, on average, five years older than TASO patients and eight years older than Uganda Cares' patients. 
Table 10 Site descriptions

\begin{tabular}{|c|c|c|c|c|c|c|}
\hline Organization & Site name & Model used & $\begin{array}{l}\text { \# total } \\
\text { clients }\end{array}$ & $\begin{array}{l}\text { \# female } \\
\text { patients }\end{array}$ & $\begin{array}{l}\text { \# male } \\
\text { clients }\end{array}$ & $\begin{array}{c}\text { Median } \\
\text { patient age, } \\
\text { yrs (IQR) }\end{array}$ \\
\hline \multirow[t]{6}{*}{ TASO } & Entebbe & Central-CDDP & 524 & 334 & 190 & $35(30-42)$ \\
\hline & Gulu & Central-CDDP & 389 & 267 & 122 & $35(29-42)$ \\
\hline & Jinja & Central-CDDP & 455 & 297 & 158 & $38(33-45)$ \\
\hline & Masindi & Central-CDDP & 220 & 154 & 66 & $36(30-42)$ \\
\hline & Rukungiri & Central-CDDP & 356 & 218 & 138 & $39(33-49)$ \\
\hline & TOTAL & & 1,944 & $1,270(65.3 \%)$ & 674 (34.7\%) & $36(30-44)$ \\
\hline \multirow{3}{*}{$\begin{array}{l}\text { Uganda } \\
\text { Cares }\end{array}$} & St. Balikudembe & Central & 129 & 69 & 60 & $33(28-40)$ \\
\hline & Nakawa Market & Central & 69 & 38 & 31 & $34(29-40)$ \\
\hline & TOTAL & & 198 & $107(54.1 \%)$ & 91 (45.9\%) & $33(28-40)$ \\
\hline $\begin{array}{l}\text { Kitovu } \\
\text { Mobile }\end{array}$ & N/A & $\begin{array}{l}\text { Community } \\
\text { Outreach }\end{array}$ & 81 & $43(53.1 \%)$ & $38(46.9 \%)$ & $4 \mid(34-49)$ \\
\hline Grand Total & & & 2,223 & $1,429(63.8 \%)$ & 809 (36.2\%) & $36(30-44)$ \\
\hline
\end{tabular}

IQR is interquartile range

\section{Service utilization, drug adherence, and retention in care}

Service utilization: Data were cleaned and complete longitudinal service histories were compiled for 2,223 ART patients who initiated treatment during the review period. At the time of ART initiation, median patient age was 36 years. Nearly two-thirds (64 percent) of the study population was female. As described in Table 10, patients attended an average of 13.3 drug refill visits during the review period, spent 49.4 days between refill visits, and received sufficient ARV medication to cover their treatment for 50.9 days.

\section{Table I I Service utilization-All sites}

\begin{tabular}{|lc|}
\hline Per-patient averages & Total \\
\hline Median time in care_days per patient (IQR) & $\mathbf{n = 2 , 2 2 3}$ \\
Mean time in care—days per patient (SE) & 80 I (74I-852) \\
No.ART refill visits (SE) & $686(270.7)$ \\
Avg. period between patient visits—days (SE) & $13.3(5.4)$ \\
Avg. days covered by drugs dispensed per visit—days (SE) & $49.4(17.2)$ \\
\hline
\end{tabular}

SE is standard error

Table 12 shows that patients of all three organizations remained in care for a similar amount of time, with averages ranging from 671 to 730 days. TASO patients on average spent 51 days between visits, attending an average of 12.7 visits in the 24 months of follow-up. At each visit, they received sufficient medication to cover an average period of 52 days before a refill was needed. Likewise, patients of Uganda Cares spent 39 days between visits, where they received 47 days-worth of medication. Kitovu Mobile appeared to 
be dispensing insufficient quantities of medication; although their patients spent an average of 35 days between visits, on average, they only received enough medication to last 30 days.

Table 12 Service utilization-by provider

\begin{tabular}{|lccc|}
\hline Per-patient averages & $\begin{array}{c}\text { Kitovu Mobile } \\
\mathbf{n = 8} \\
\mathbf{n}(\%)\end{array}$ & $\begin{array}{c}\text { UC } \\
\mathbf{n}=198\end{array}$ & $\begin{array}{c}\text { TASO } \\
\mathbf{n}=1,944\end{array}$ \\
\hline Days retained in care (SE) & $815(24.9)$ & $812(21.2)$ & $\mathbf{n}(\%)$ \\
Mean days-in-care, per-patient (SE) & $729.8(224)$ & $671.4(297.7)$ & $686.4(269.5)$ \\
No.ART refill visits attended (SE) & $21.3(6.7)$ & $17.5(7.7)$ & $12.7(4.7)$ \\
Avg period between patient visit-days (SE) & $35.0(11.0)$ & $39.0(16.4)$ & $51.0(16.9)$ \\
Avg. drugs dispensed per visit—days (SE) & $29.5(9.3)$ & $46.5(19.2)$ & $52.2(8.1)$ \\
\hline
\end{tabular}

Drug adherence: Though patients were typically dispensed quantities of medication that were sufficient to last until their next refill visit, treatment gaps were common. Table 13 shows that 40 percent of patients had one or more gaps in treatment lasting at least 45 days, and many patients had multiple gaps. Gap duration varied widely, ranging from 48 to 613 days, with patients spending an average of 112.0 days (95\% CI: 107.9-116.1) away from the health facility before returning to care. Based on the available data, it was not possible to determine whether patients obtained any medication from an external source during treatment gaps. After 24 months of follow-up, 54.5 percent of patients had received sufficient ARV medication to cover at least 95 percent of their expected treatment. In other words, based on available documentation of dispensed drugs, half of all clients attending the TASO, UC, and Kitovu Mobile sites surveyed did not receive sufficient medication to for their treatment.

Table 13 Treatment gaps and drug adherence-all sites

\begin{tabular}{|lcc|}
\hline Outcome $(\mathbf{n}=\mathbf{2 , 2 2 3})$ & & $95 \% \mathrm{Cl}$ \\
\hline Patients $\mathbf{w} /$ at least I treatment gap $>45$ days & $896(40.3 \%)$ & $38.2 \%-42.3 \%$ \\
Mean treatment gap duration—number of days (SE) & $112(2.1)$ & $107.9-116.1$ \\
Patients achieving $\geq 95 \%$ drug adherence & $1,21 \mathrm{I}(54.5 \%)$ & $52.4 \%-56.5 \%$ \\
\hline
\end{tabular}

$\mathrm{Cl}$ is confidence interval

Table 14 shows that approximately 40 percent of patients from all providers had at least one gap in treatment that exceeded 45 days. The mean duration of these gaps ranged from 89 days at Kitovu Mobile to 113.6 days at TASO. However, only 18.5 percent of Kitovu Mobile patients attained greater than 95 percent drug adherence during their time in treatment. At TASO and Uganda Cares sites, 54 percent and 71 percent of patients, respectively, reached this benchmark. 
Table 14 Treatment gaps and drug adherence-by provider

\begin{tabular}{|lccc|}
\hline Outcome & Kitovu Mobile & UC & TASO \\
& $\mathbf{n = 8 \text { I }}$ & $\mathbf{n = 1 9 8}$ & $\mathbf{n = 1 , 9 4 4}$ \\
\hline Patients with $\geq 1$ treatment gap & $32(39.5 \%)$ & $85(42.9 \%)$ & $778(40 \%)$ \\
Mean treatment gap duration— number of days (SE) & $88.7(50.5)$ & $108.2(67.7)$ & $113.6(62.2)$ \\
Patients achieving $\geq 95 \%$ drug adherence & $15(18.5 \%)$ & $140(70.7 \%)$ & $1,059(54.5 \%)$ \\
\hline
\end{tabular}

Retention: There were four possible outcomes relating to the retention of ART patients: death, transfer to another facility, loss to follow-up, and completion of the follow-up period. In the clinic records, only death and patient transfer were documented. Patients who were in default as of December 31, 2012 were considered lost to follow-up unless the facility records specified that the patient had transferred to another facility or had died. Table 15 shows that of 2,223 patients reviewed at all sites, 1,667 patients (75 percent) were actively retained in care after 24 months, 16 patients ( 0.7 percent) had transferred to another facility, and six patients ( 0.3 percent) had died. The remaining 24 percent of patients (534) had defaulted on their care without resuming treatment by December 31,2012, and had no final outcome documented in their records; all were deemed lost to follow-up. However the caveat must be made that the quality and completeness of final patient outcome records varied by organization and facility. Therefore, it was not possible to ascertain whether any lost to follow-up patients may have actually died or transferred to another facility. From the perspective of improving drug adherence, facilities should consider any defaulting patients to be in need of support until the patient resumes care, or an ultimate outcome can be determined.

Kitovu Mobile had the most complete records on client outcomes. As shown in Table 15, by the end of the data collection period, 17 Kitovu Mobile patients had left the cohort. Of these, six had died, and another six had transferred to other facilities. The remaining five patients had no recorded outcome, thus they were categorized as lost to follow-up. Records from TASO indicated that 479 patients were not retained in care. Of these, records indicated that only 10 had transferred to other facilities and no data on any patient deaths were recorded. As the remaining 469 had no documented outcomes, we categorized all of them as lost to follow-up. UC provided no documented outcomes for any patients who were not retained, therefore all 60 non-retained patients are treated as lost to follow-up.

\section{Table I5 Outcomes at 24 months}

\begin{tabular}{|lcccc|}
\hline Ultimate patient outcomes & $\begin{array}{c}\text { Kitovu Mobile } \\
\mathbf{n = 8}\end{array}$ & $\mathbf{n C}$ & TASO & All sites \\
& $\mathbf{n}(\%)$ & $\mathbf{n}=198$ & $\mathbf{n}=1,944$ & $\mathbf{N}=\mathbf{2 , 2 2 3}$ \\
Lost to follow-up & $5(6.2 \%)$ & $60(30.3 \%)$ & $\mathbf{n}(\%)$ & $\mathbf{N}(\%)$ \\
Transfer out & $6(7.4 \%)$ & 0 & $10(24.1 \%)$ & $534(24.0 \%)$ \\
Retained & $64(79.0 \%)$ & $138(69.7 \%)$ & $1,465(75.4 \%)$ & $16(0.7 \%)$ \\
Died & $6(7.4 \%)$ & 0 & 0 & $6(0.67 \%)$ \\
\hline
\end{tabular}

Table 16 shows that retention was highest during the first six months after a patient's initiation onto ART and lowest at 24 months. At the bottom of Table 16, the data on loss to follow-up shows that for all sites, 
patients who were ultimately lost to follow-up spent a median of 217 days in care, and completed an average of five visits. Nearly a quarter of all patients (23.7 percent, $n=127)$ were lost to follow-up within 30 days of initiating ART; 15.7 percent $(n=84)$ completed only one visit before dropping out. Nearly a quarter of all loss to follow-up at TASO (24.3 percent) and UC (21.7 percent), occurred within the first 30 days after initiation; 13.3 percent of UC patients, and 16.2 percent of TASO patients completed only their initial ART visit before dropping out. Kitovu Mobile lost no patients after the first visit, nor within the first 30 days. A chi-square test indicates that there was significant variability in patient retention between the three providers $(\mathrm{p}<0.0001)$.

\section{Table 16 Retention and loss to follow-up by duration}

\begin{tabular}{|c|c|c|c|c|}
\hline Client retention & $\begin{array}{c}\text { Kitovu Mobile } \\
\begin{array}{c}n=8 \\
n(\%)\end{array}\end{array}$ & $\begin{array}{c}\text { UC } \\
n=198 \\
n(\%)\end{array}$ & $\begin{array}{c}\text { TASO } \\
n=1,944 \\
n(\%)\end{array}$ & $\begin{array}{c}\text { All sites } \\
N=2,223 \\
N(\%)\end{array}$ \\
\hline No. patients retained after 6 months & 77 (95.1\%) & 169 (85.4\%) & ।,727 (88.8\%) & I,973 (88.8\%) \\
\hline No. patients retained after 12 months & $76(93.8 \%)$ & $161(81.3 \%)$ & I,632 (84.0\%) & $1,869(84.1 \%)$ \\
\hline No. patients retained after 18 months & 72 (88.9\%) & 147 (74.2\%) & ।,572 (80.9\%) & I,691 (76.1\%) \\
\hline No. patients retained after 24 months & 70 (86.4\%) & $138(69.7 \%)$ & ।,475 (75.9\%) & I,683 (75.7\%) \\
\hline Loss to follow-up & $\begin{array}{c}\text { Kitovu Mobile } \\
n=5\end{array}$ & $\begin{array}{c}\text { UC } \\
n=60\end{array}$ & $\begin{array}{l}\text { TASO } \\
n=469\end{array}$ & $\begin{array}{l}\text { All sites } \\
N=534\end{array}$ \\
\hline Median time to loss to follow-up-days (IQR) & $374(224-495)$ & $286.5(4-473)$ & $210(31-465)$ & $217(34-467)$ \\
\hline Median no. visits completed (IQR) & $7(6-12)$ & $5(3-12.5)$ & $5(2-9)$ & $5(2-10)$ \\
\hline Patients lost to follow-up $\leq 30$ days (\%) & 0 & $13(21.7 \%)$ & II 4 (24.3\%) & $127(23.8 \%)$ \\
\hline Patients lost to follow-up after I visit (\%) & 0 & $8(13.3 \%)$ & $76(16.2 \%)$ & 84 (15.7\%) \\
\hline
\end{tabular}

Figure 5 depicts retention in care at six-month intervals for each of the three organizations. Retention in ART care at 24 months was 86 percent at Kitovu Mobile, 75.9 percent at TASO, and 70 percent at UC. This variation in retention rates among the three organizations is also seen at the 6,12 , and 18 months intervals.

Factors associated with loss to follow-up: Using chi-squared tests, we examined associations between loss to follow-up and other related factors. The most prominent risks for loss to follow-up were sex, being below median age, and primary service location. Significant associations are detailed in Table 17 . Due to the much larger size of TASO's cohort, most associations were observed among TASO clients. Males were 1.6 times more likely than women to be lost to follow-up ( $<0.0001)$; patients under 36 years of age were 1.3 times more likely to be lost to follow-up, though significance was marginal $(p=0.036)$. Chi-squared tests for homogeneity indicated that there were significant differences in loss to follow-up between the five TASO facilities that we surveyed $(\mathrm{p}=0.001)$. 
Figure 5 Comparison of retention in care at the three ASOs

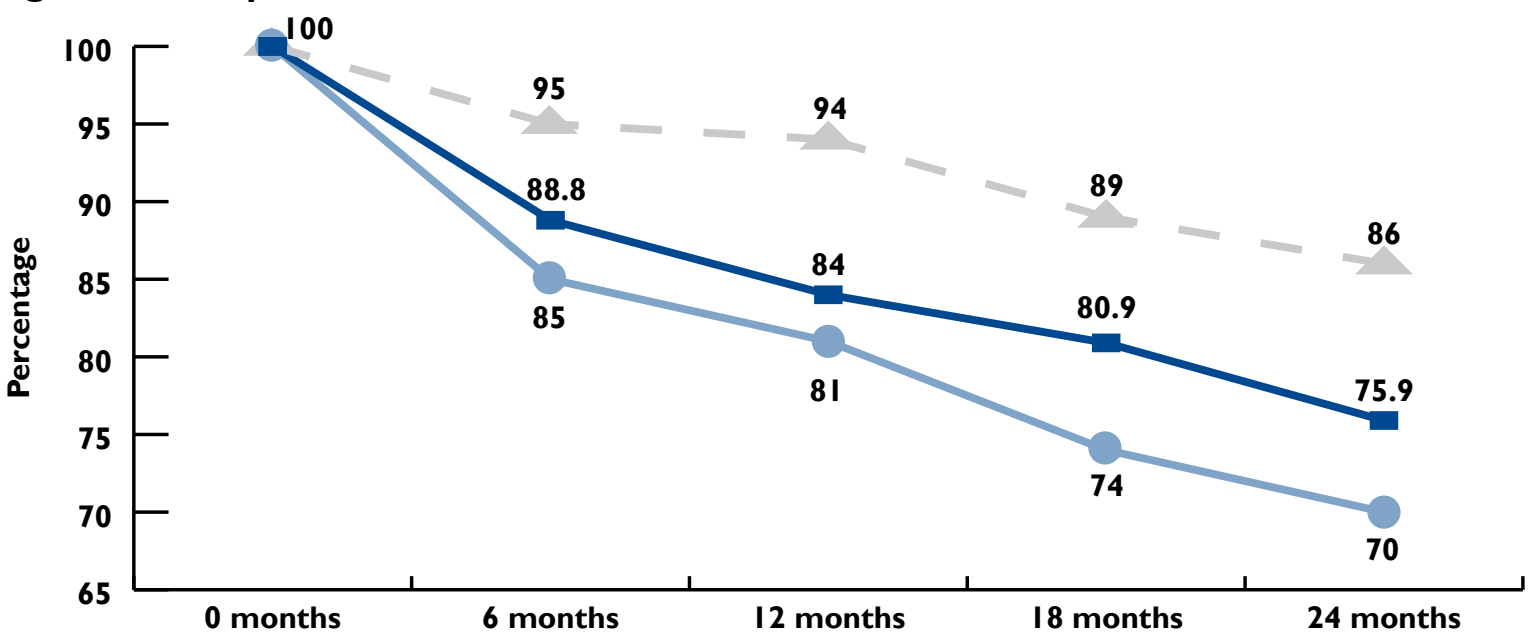

Kitovu Mobile $(n=81)-$ TASO $(n=1,944)-O$ UC $(n=198)$

Table I 7 Factors associated with loss to follow-up

\begin{tabular}{|c|c|c|c|}
\hline Bivariate associations & OR & p-value & $95 \% \mathrm{Cl}$ \\
\hline \multicolumn{4}{|l|}{$\operatorname{Sex}(n=1,944)$} \\
\hline Female & 1.00 & - & - \\
\hline Male & 1.61 & $<0.0001 * *$ & {$[1.313-2.015]$} \\
\hline \multicolumn{4}{|l|}{ Age $(n=1,944)$} \\
\hline$\geq 36$ years & 1.00 & - & - \\
\hline$<36$ years & 1.25 & $0.036 *$ & {$[1.014-1.538]$} \\
\hline \multicolumn{4}{|l|}{ Location of primary clinic } \\
\hline \multicolumn{4}{|l|}{$U C(n=198)$} \\
\hline St. Balikudembe & 1.00 & & \\
\hline Nakawa Market & 1.93 & 0.056 & {$[0.972-3.826]$} \\
\hline TASO (5 locations; $n=1,944)$ & & $0.001 * *$ & \\
\hline Rukungiri & 1.00 & - & - \\
\hline Gulu & 1.01 & 0.97 & {$[0.703-1.443]$} \\
\hline Jinja & 1.26 & 0.17 & \\
\hline Masindi & 1.24 & 0.29 & \\
\hline Entebbe & 1.75 & $0.0006 * *$ & \\
\hline
\end{tabular}


Associations between task- and site-shifting and patient retention in care: Only TASO provided data from ART services delivered to patients through both their central facilities and community-based service points. We assessed relationships between CDDP attendance and patient retention rates using multiple logistic regression. Table 18 describes CDDP utilization among the subset of 1,757 TASO patients who were sufficiently stabilized in their care, and thus eligible to receive services at a CDDP (i.e., had completed at least four refill visits). One third were male, and the median age was 37 years; 1,636 patients attended at least one CDDP-based refill visit, while 121 patients attended only facility-based refill visits. Age and mean number of refill visits were significantly associated with ever using a CDDP: older patients were significantly more likely to have ever visited a CDDP; patients who ever visited a CDDP completed significantly more refill visits.

Table 18 Bivariate analysis of TASO patient CDDP utilization

\begin{tabular}{|c|c|c|}
\hline Among stable TASO patients & $\begin{array}{c}\text { No CDDP attendance } \\
\text { n (row \%) }\end{array}$ & $\begin{array}{c}\text { Attended CDDP } \\
\text { n (row \%) }\end{array}$ \\
\hline $\begin{array}{l}\text { Stable patients } \\
\text { (\# visits } \geq 4, n=1,757)\end{array}$ & $121(6.9)$ & $1,636(93.1)$ \\
\hline \multicolumn{3}{|l|}{ Sex } \\
\hline Male $(n=586: 33 \%)$ & $36(6.1)$ & $550(93.9)$ \\
\hline Female $(n=1,|7|: 67 \%)$ & $85(7.3)$ & I,086 (92.7) \\
\hline \multicolumn{3}{|l|}{ Age } \\
\hline$<37$ years $(n=808: 49.1 \%)^{*}$ & $69(8.5)$ & $739(91.5)$ \\
\hline$\geq 37$ years $(n=949: 50.9 \%$ )* & $52(5.5)$ & $897(94.5)$ \\
\hline \multicolumn{3}{|l|}{ Location of primary clinic } \\
\hline Entebbe $(n=438)$ & $26(5.9)$ & $412(94.0)$ \\
\hline Gulu $(n=364)$ & $28(7.7)$ & $336(92.3)$ \\
\hline Jinja $(n=416)$ & $36(8.7)$ & $380(91.3)$ \\
\hline Masindi $(n=215)$ & $14(6.5)$ & $201(93.5)$ \\
\hline Rukungiri $(n=324)$ & $17(5.2)$ & $307(94.8)$ \\
\hline \multicolumn{3}{|c|}{ Mean number of drug refill visits completed } \\
\hline 12 months $[95 \% \mathrm{Cl}]^{* *}$ & $6.5[6.2-6.9]$ & $7.3[7.3-7.4]$ \\
\hline 18 months $[95 \% \mathrm{Cl}]^{* *}$ & $8.1[7.5-8.6]$ & $10.2[10.1-10.2]$ \\
\hline Total $[95 \% \mathrm{Cl}]^{* *}$ & $10.0[9.1-10.9]$ & $13.8[\mid 3.7-14.0]$ \\
\hline \multicolumn{3}{|l|}{ Proportions of patients retained } \\
\hline 12 months $(\%)^{* *}$ & $67 / 121(55.3)$ & I,559/I $636(95.3)$ \\
\hline 18 months (\%)** & $58 / 121(47.4)$ & $1,510 / 1636(92.3)$ \\
\hline Overall (\%)** & $52 / 121(42.3)$ & $1,419 / 1636(86)$ \\
\hline
\end{tabular}

*Significant difference between groups who did/did not attend any CDDP-based service $(\mathrm{p}<0.05)$.

**Significant difference between groups who did/did not attend any CDDP-based service $(p<0.00 \mathrm{I})$.

Stable TASO patients who received any services through a CDDP were significantly more likely to be retained at 12 (OR: 16.3; 95\% CI: 10.3-25.9) and 18 months (OR: 13.0; 95\% CI: 8.5-20.0) than 
patients who attended no CDDP-based visits. To examine whether intensity of exposure to CDDPbased services affected rates of retention and drug coverage, we first attempted to account for behavioral biases arising from differences in refill visit attendance between clinic-only clients and those who utilized CDDPs. Via Student's t-test, we determined that among the subset of 1,568 clients who attended at least 10 refill visits, any differences in the average total number of refill visits that each client completedregardless of whether they had attended a CDDP $(n=1,509)$, or received only clinic-based services $(\mathrm{n}=59)$-were not significant $(\mathrm{p}>0.34)$. Among this subset, 111 were lost to follow-up, three had transferred, and 1,454 were retained after 24 months.

Among this subset of clients, we constructed a three-level exposure variable. As this population had considerable variability in their total number of refill visits attended $(\min =10$, $\max =31)$, we based the exposure variable on the proportion of total refill visits that an individual client completed at a CDDP. For this sub-population, median CDDP exposure was 66 percent. Thus, the 749 clients who attended fewer than 66 percent of their total visits at a CDDP were classified as having low CDDP exposure, while the 760 clients who attended more than 66 percent of their total refill visits at a CDDP were classified as high-exposure. The third level of exposure comprised 59 clients who had no CDDP exposure.

Finally, we included this exposure variable in a logistic regression model controlling for age, sex, primary service location, and total number of visits completed during 24 months of follow-up. This analysis indicates that clients with high exposure to CDDP services were significantly more likely to be retained after 24 months than clients with low CDDP exposure (OR: 4.15, 95\% CI: 1.83-9.41). However, differences in retention between low-exposure and no-exposure groups were not significant $(\mathrm{OR}=1.95$, 95\%CI: 0.89-4.25). Among the 1,454 TASO clients who completed at least 10 visits and were retained after 24 months, CDDP utilization had no significant association with achieving 95 percent drug coverage during their time in care.

\section{HEALTH SYSTEMS OUTCOMES}

This section presents findings regarding the implications of task-shifting on two key health systems outcomes-quality of care and cost of care.

\section{Observed performance of providers}

Table 19 shows that the vast majority of the sessions observed were repeat ART visits. The type of provider observed varied by clinic, reflecting the distribution of providers who conducted one-on-one consultation sessions with patients, since only clinical- or counseling-type of consultation sessions were selected for observation. For Kitovu Mobile the majority of sessions observed were conducted by a nurse; at UC-MOH the majority was split between the clinical officer and nurse; at UC-S the majority was split between doctors, clinical officers, and HIV medics; at TASO-CDDP the majority of sessions observed were conducted by the counselor; while at TASO-C the majority were conducted by the clinical officer. 
Table 19 Characteristics of service sessions observed for CPI

\begin{tabular}{|c|c|c|c|c|c|}
\hline & $\begin{array}{l}\text { Kitovu } \\
\text { Mobile }\end{array}$ & UC-MOH & UC-S & $\begin{array}{l}\text { TASO- } \\
\text { CDDP }\end{array}$ & TASO-C \\
\hline Number of health facility sites & 4 & 6 & 3 & 10 & 5 \\
\hline Number of sessions observed & 203 & 310 & 136 & 498 & 271 \\
\hline \multirow[t]{2}{*}{ Average number of sessions per site } & 51 & 52 & 45 & 50 & 54 \\
\hline & $\%$ & $\%$ & $\%$ & $\%$ & $\%$ \\
\hline \multicolumn{6}{|l|}{ Type of patient visit } \\
\hline First visit & 0.5 & 1.6 & 0.0 & 0.0 & 6.3 \\
\hline Repeat visit & 99.5 & 98.4 & 100.0 & 100.0 & 93.7 \\
\hline \multicolumn{6}{|l|}{ Type of provider } \\
\hline Doctor & 0.0 & 13.9 & 26.6 & 1.0 & 5.5 \\
\hline Clinical officer & 5.4 & 42.3 & 28.8 & 14.9 & 65.7 \\
\hline Nurse & 94.6 & 37.7 & 8.9 & 10.2 & 3.7 \\
\hline Counselor & 0.0 & 0.0 & 0.0 & 66.5 & 16.6 \\
\hline Lab tech & 0.0 & 0.0 & 0.0 & 0.6 & 1.5 \\
\hline Lab assistant & 0.0 & 0.0 & 0.0 & 2.4 & 3.3 \\
\hline Pharmacy worker & 0.0 & 0.0 & 0.0 & 0.0 & 2.6 \\
\hline Counseling assistant & 0.0 & 0.0 & 0.0 & 1.2 & 0.0 \\
\hline $\mathrm{CHW}$ & 0.0 & 0.0 & 0.0 & 0.6 & 0.0 \\
\hline Expert client & 0.0 & 0.0 & 0.0 & 2.4 & 0.4 \\
\hline HIV medic & 0.0 & 6.1 & 35.5 & 0.0 & 0.4 \\
\hline Other & 0.0 & 0.0 & 0.0 & 0.2 & 0.0 \\
\hline
\end{tabular}

Table 20 shows that while the performance of assessment of the patients was 66 percent to 74 percent and the performance of addressing complaints was 70 percent to 89 percent, there was a relatively low performance with regard to adherence counseling ( 42 percent to 55 percent), and counseling about side effects ( 30 percent to 42 percent). To examine how the quality of care varies with the level of task-shifting we compared the quality of care data between the facility-based clinics (TASO-C, UC-MOH, and UC-S) and the community-based clinics (Kitovu Mobile and TASO-CDDP). Table 20 shows that the providers at the facility-based clinics performed significantly better in addressing the patients' complaints compared to community-based settings ( $\mathrm{p}<0.01$ ). However, there was no significant difference between these two types of clinics in the performance of the other key ART care tasks ( $p>0.05)$. 


\section{Table 20 Comparison of performance of providers between community-based and facility-based clinics (CPI observations)}

\begin{tabular}{|c|c|c|c|}
\hline & $\begin{array}{c}\text { Community- } \\
\text { based } \\
n=696 \\
\text { Prop }\end{array}$ & $\begin{array}{c}\text { Facility- } \\
\text { based } \\
n=714 \\
\text { Prop }\end{array}$ & p-value \\
\hline Assessment of patient (weighing; asking about complaints; exploring issues) & 0.66 & 0.74 & $>0.05$ \\
\hline $\begin{array}{l}\text { Adherence counseling (asking about: missed dose, challenges in adherence, } \\
\text { and family support; discussing adherence on ARVs received today) }\end{array}$ & 0.42 & 0.55 & $>0.05$ \\
\hline $\begin{array}{l}\text { Side effects counseling (asking about side effects; discussing signs and } \\
\text { symptoms of side effects and what should be done in case of side effects) }\end{array}$ & 0.30 & 0.42 & $>0.05$ \\
\hline $\begin{array}{l}\text { Addressing complaints (providing solutions or referrals for each of the } \\
\text { complaints raised by patient) }\end{array}$ & 0.70 & 0.89 & $<0.01$ \\
\hline
\end{tabular}

In summary, while there was no significant difference between the two levels of task-shifting in provider performance of most of the key ART care tasks observed, the performance of providers in addressing patients' complaints was significantly lower at the community-based than at the facility-based clinics.

As mentioned before, Uganda Cares has a unique type of LHW, the HIV medic that was created for task-shifting of ART care. Indeed as Table 21 shows, among the sessions observed the HIV medic was the provider in 67 sessions while in 382 sessions the providers were other health workers: doctors $(\mathrm{n}=$ $82)$, clinical officers $(n=167)$ and nurses $(n=133)$. Comparison of performance shows that there was no statistically significant difference between the performance of HIV medics and the other health workers in any of the key tasks in ART care $(\mathrm{p}>0.05)$.

\section{Table 2 I Comparison of performance of tasks at UC clinics by type of HW} (CPI observations)

\begin{tabular}{|c|c|c|c|}
\hline & $\begin{array}{l}\text { HIV medics } \\
\qquad \begin{array}{l}n=67 \\
\text { Prop }\end{array}\end{array}$ & $\begin{array}{l}\text { Other HWs } \\
\begin{array}{c}n=382 \\
\text { Prop }\end{array}\end{array}$ & p-value \\
\hline $\begin{array}{l}\text { Assessment of patient (weighing; asking about complaints; exploring } \\
\text { issues) }\end{array}$ & 0.85 & 0.93 & $>0.05$ \\
\hline $\begin{array}{l}\text { Adherence counseling (asking about: missed dose, challenges in } \\
\text { adherence, and family support; discussing adherence on ARVs } \\
\text { received today) }\end{array}$ & 0.60 & 0.62 & $>0.05$ \\
\hline $\begin{array}{l}\text { Side effects counseling (asking about side effects; discussing signs } \\
\text { and symptoms of side effects and what should be done in case of } \\
\text { side effects) }\end{array}$ & 0.32 & 0.35 & $>0.05$ \\
\hline $\begin{array}{l}\text { Addressing complaints (providing solutions or referrals for each of } \\
\text { the complaints raised by patient) }\end{array}$ & 0.96 & 0.98 & $>0.05$ \\
\hline
\end{tabular}




\section{Time spent}

The calculation of time spent with each patient was based on the provider of the main consultation session that, we assumed, had to see every patient attending the clinic. As documented in the facility audits, this provider differed across the five models within the three ASOs described earlier. ${ }^{7}$ Based on this assumption, the time available for each patient with the provider of the main consultation ranged from 9 to 27 minutes (Table 22). While no gold standard exists, it is our opinion that patients spent sufficient time with providers of main consultation sessions within each of these five models.

Table 22 Time spent on clinical review (Facility audits)

\begin{tabular}{|c|c|c|c|c|c|}
\hline & $\begin{array}{l}\text { Kitovu } \\
\text { Mobile }\end{array}$ & UC-MOH & UC-S & $\begin{array}{l}\text { TASO- } \\
\text { CDDP }\end{array}$ & TASO-C \\
\hline $\begin{array}{l}\text { a. Median clinic duration from open to close } \\
\text { (min) }\end{array}$ & 288 & 392 & 465 & 227 & 549 \\
\hline b. Total patients seen on day of audit & 196 & 266 & 283 & 561 & 594 \\
\hline $\begin{array}{l}\text { c. Cadre responsible for the main } \\
\text { consultation session }\end{array}$ & $\begin{array}{c}\text { Nurse }(n= \\
\text { 13) }\end{array}$ & $\begin{array}{l}\text { Dr. or CO } \\
(n=9)\end{array}$ & $\begin{array}{l}\text { Dr. or CO } \\
(n=6)\end{array}$ & $\begin{array}{l}\text { Counselor } \\
(\mathrm{n}=22)\end{array}$ & $\begin{array}{l}\text { Dr. or CO } \\
(n=27)\end{array}$ \\
\hline $\begin{array}{l}\text { d. Number of patients per provider of the } \\
\text { main consultation session }(=b \div c)\end{array}$ & 15 & 30 & 47 & 26 & 20 \\
\hline $\begin{array}{l}\text { e. Average minutes per client for clinical } \\
\text { review }(=\mathrm{a} \div \mathrm{d})\end{array}$ & 19 & 13 & 10 & 9 & 27 \\
\hline
\end{tabular}

Table 23 shows that the overall time spent by each patient at the clinic ranged from 48 minutes at UC-S to 3 hours and 45 minutes at Kitovu Mobile. The time that was spent on waiting ranged from 33 minutes at UC-S to 3 hours and 15 minutes at Kitovu Mobile. The contact time with clinic staff appears to be within the same order of magnitude across the five models (15 to 29 minutes).

Table 23 Median time spent per patient (Time-motion study)

\begin{tabular}{|c|c|c|c|c|c|}
\hline & $\begin{array}{l}\text { Kitovu } \\
\text { Mobile }\end{array}$ & UC-MOH & UC-S & $\begin{array}{l}\text { TASO- } \\
\text { CDDP }\end{array}$ & TASO-C \\
\hline Median overall time spent at clinic (min) & 225 & 128 & 48 & 200 & 221 \\
\hline $\begin{array}{l}\text { Median total contact time with all clinic staff } \\
\text { (min) }\end{array}$ & 15 & 20 & 18 & 18 & 29 \\
\hline Median total waiting time (min) & 205 & 102 & 33 & 170 & 185 \\
\hline $\begin{array}{l}\text { Percentage of total patient time spent with } \\
\text { a clinic staffer }\end{array}$ & $6.7 \%$ & $15.6 \%$ & $37.5 \%$ & $9 \%$ & $13.1 \%$ \\
\hline
\end{tabular}

\footnotetext{
${ }^{7}$ The five models are as follows: TASO has two models-TASO Central (TASO-C), which is facility-based, and TASO Community Drug Distribution Points (TASO-CDDP), which is a community outreach program. Uganda Cares has two models, both of which are facility-based-Uganda Cares Stand-alone (UC-S) which is operated at clinics owned by UC, and Uganda Cares MOH (UC-MOH), which is operated at MOH clinics. Kitovu Mobile has only one model — a community outreach program.
} 
Thus, in most of the models patients spent a long time at the clinic, most of which was spent waiting rather than in contact with providers. However, it is notable that UC-S has much shorter patient waiting times than other models and is worth exploring further to identify possible best practices in time management at UC-S that can be replicated.

\section{Cost per visit and cost per client}

Table 24 summarizes the 2012 total annual ART-related expenditure, the cost per client visit, and annual cost per client in USD. Table 24 also shows the average number of visits per client per year across the three models. In particular, clients at Kitovu Mobile made an average of nine visits to the Kitovu Mobile outreach locations for ART care, while TASO clients made an average of about five visits, and UC clients made about seven visits, annually. The average cost per visit was $\$ 38$ for UC, $\$ 45$ for Kitovu Mobile, and $\$ 74$ for TASO clients. The average annual cost per client was $\$ 404$ for Kitovu Mobile, $\$ 332$ for TASO, and $\$ 257$ for UC clients. The average cost per client for all three organizations was $\$ 331$ and varied across the four TASO centers and four UC clinics.

Table 24 Average cost per visit and annual cost per client across the 3 models (20 I 2)

\begin{tabular}{|c|c|c|c|c|c|c|c|}
\hline Model & Location & $\begin{array}{c}\text { Total } \\
\text { expenditure } \\
\text { (USD) }\end{array}$ & $\begin{array}{l}\text { Total } \\
\text { ART } \\
\text { clients }\end{array}$ & $\begin{array}{l}\text { Total } \\
\text { ART } \\
\text { visits }\end{array}$ & $\begin{array}{c}\text { Average } \\
\text { annual } \\
\text { visits/ } \\
\text { client }\end{array}$ & $\begin{array}{l}\text { Average } \\
\text { cost per } \\
\text { ART visit } \\
\text { (USD) }\end{array}$ & $\begin{array}{c}\text { Annual } \\
\text { cost per } \\
\text { ART } \\
\text { client } \\
\text { (USD) }\end{array}$ \\
\hline $\begin{array}{l}\text { Kitovu } \\
\text { Mobile }\end{array}$ & $\begin{array}{l}\text { Rural- } \\
\text { Southwestern region }\end{array}$ & $\$ 560,756$ & $\mathrm{I}, 387$ & 12,510 & 9.0 & $\$ 45$ & $\$ 404$ \\
\hline \multirow[t]{5}{*}{ TASO } & Rural—Gulu & $\$ 2,094,695$ & 6,969 & 28,654 & 4.1 & $\$ 73$ & $\$ 301$ \\
\hline & Rural—Rukungiri & $\$ 1,944,096$ & 4,602 & 27,693 & 6.0 & $\$ 70$ & $\$ 422$ \\
\hline & Semi-Urban-Jinja & $\$ 1,969,940$ & 5,454 & 32,233 & 5.9 & $\$ 61$ & $\$ 361$ \\
\hline & $\begin{array}{l}\text { Semi-Urban- } \\
\text { Entebbe }\end{array}$ & $\$ 1,744,231$ & 6,329 & 23,461 & 3.7 & $\$ 74$ & $\$ 276$ \\
\hline & OVERALL COST & $\$ 7,752,962$ & 22,814 & || $2,04 \mid$ & 4.8 & $\$ 69$ & $\$ 332$ \\
\hline \multirow[t]{5}{*}{$\begin{array}{l}\text { Uganda } \\
\text { Cares }\end{array}$} & $\begin{array}{l}\text { Urban- } \\
\text { St. Balikudembe }\end{array}$ & $\$ 791,009$ & 2,498 & 12,636 & 5.1 & $\$ 63$ & $\$ 317$ \\
\hline & $\begin{array}{l}\text { Urban- } \\
\text { Nakawa Market }\end{array}$ & $\$ 190,222$ & 530 & 6,076 & 11.5 & $\$ 31$ & $\$ 359$ \\
\hline & Rural—Lyantonde & $\$ 272,84 I$ & 1,250 & 10,345 & 8.3 & $\$ 26$ & $\$ 218$ \\
\hline & Rural—Kalisizo & $\$ 274,717$ & 1,669 & 11,420 & 6.8 & $\$ 24$ & $\$ 165$ \\
\hline & OVERALL COST & $\$ 1,528,789$ & 5,947 & 40,477 & 6.8 & $\$ 38$ & $\$ 257$ \\
\hline
\end{tabular}

Table 25 shows the distribution of costs for each model. For Kitovu Mobile, ARV supplies comprised 47 percent of the total cost, personnel was 25 percent, operational expenses were 15 percent, 11 percent was used to procure non-ARV drugs, and 2 percent was paid to ART-related lab services. TASO's cost distribution was fairly similar to Kitovu Mobile, with 44 percent attributed to ARV drugs, 21 percent attributed to personnel, 15 percent attributed to non-ARV drugs, 14 percent attributed to administrative expenses, and 6 percent spent on lab tests. UC's cost distribution was different from both Kitovu Mobile 
and TASO. In particular, UC's ART drug-related expenses accounted for 66 percent of the total cost, followed by lab tests (13 percent), personnel ( 9 percent), administrative ( 8 percent), and non-ARV drugs (4 percent).

Table 25 Cost distribution across 5 cost components (20 I 2)

\begin{tabular}{|lcccccc|}
\hline Model & $\begin{array}{c}\text { Personnel } \\
\$(\%)\end{array}$ & $\begin{array}{c}\text { ARV } \\
\text { supplies } \\
\$(\%)\end{array}$ & $\begin{array}{c}\text { Non-ARV } \\
\text { medical } \\
\text { supplies } \\
\$(\%)\end{array}$ & $\begin{array}{c}\text { Laboratory } \\
\$(\%)\end{array}$ & $\begin{array}{c}\text { Administrative } \\
\text { costs } \\
\$(\%)\end{array}$ & $\begin{array}{c}\text { Total US } \$ \\
\$(\%)\end{array}$ \\
\hline Kitovu & $\$ 140,529$ & $\$ 260,641$ & $\$ 59,439$ & $\$ 13,150$ & $\$ 86,996$ & $\$ 560,756$ \\
Mobile & $25.1 \%$ & $46.5 \%$ & $10.6 \%$ & $2.3 \%$ & $15.5 \%$ & $100.0 \%$ \\
TASO & $\$ 1,655,930$ & $\$ 3,399,418$ & $\$ 1,145,544$ & $\$ 477,737$ & $\$ 1,074,333$ & $\$ 7,752,962$ \\
& $21.4 \%$ & $43.8 \%$ & $14.8 \%$ & $6.2 \%$ & $13.9 \%$ & $100.0 \%$ \\
Uganda & $\$ 135,106$ & $\$ 1,007,376$ & $\$ 65,441$ & $\$ 199,366$ & $\$ 121,500$ & $\$ 1,528,789$ \\
Cares & $8.8 \%$ & $65.9 \%$ & $4.3 \%$ & $13.0 \%$ & $7.9 \%$ & $100.0 \%$ \\
\hline
\end{tabular}




\section{LIMITATIONS}

\section{OVERALL STUDY LIMITATIONS}

In order to infer conclusions about the effect of task-shifting on health outcomes, an ideal study design should have involved at least a two-arm comparison between task-shifted and not task-shifted services. In addition, cost data should have included financial, economic, and opportunity costs incurred by both providers and clients. To date at least three meta-analyses (referenced in the discussion) have been published on the subject of ART task-shifting. The inclusion criteria for studies into these analyses have tended to be: a randomized control trial comparing one provider against another of a different level of qualification or comparing one level of health facility; or a cohort design with a baseline measurement followed by the introduction of a task-shifting approach and then an endline measurement. However, our study is a descriptive study examining different models of task-shifting that were designed, not for comparison, but for increasing access to ARVs by delegating some tasks to lower level HWs and lower level health care settings. The aim of our study was to capture, to the extent possible, the real life situation under which ASOs are making decisions to increase access to ART using less costly, more available space and personnel and what sort of outcomes can be associated with these decisions. In this effort we encountered another limitation-the retrospective services statistics and financial data had not been kept in a manner intended for a study but rather for practical use in routine service delivery and accounting respectively. This resulted in delays in the study timeline due to the need for data cleaning and data-reentry in some cases. For this reason, this study which examined data for the period 2010 to 2012 is being published several years later and may not adequately influence the fast-moving global trend, as shown in the literature, towards adoption of ART task-shifting as a standard of care. However, we are hopeful that our data will be useful in the continued refinement of task-shifting.

In addition, there are inherent organizational and structural differences in the task-shifting services studied. Based on the descriptions in the programs annual reports, five fairly distinct models of taskshifting emerged. However, given the differences in structures, staffing, and other resources available in each of these models, it may not be prudent to subject them to a rigorous comparison.

\section{LIMITATIONS OF MANAGEMENT INFORMATION SYSTEM DATA}

A number of limitations should be noted in interpreting the findings on retention and ARV adherence that were derived from patient records. The quality of the data contained in the patient health records varied widely by facility and organization, resulting in several important gaps:

1. The records of many patients were incomplete. Records from the histories of 238 TASO patients and six Kitovu mobile patients were missing essential data. These patient histories were excluded from analysis. In the case of Uganda Cares, the majority of service records were incomplete. Therefore, data collectors sampled a small portion of Uganda Cares' entire management information system (MIS) database to construct complete patient histories. 
2. We found considerable variability in the quantity of drugs dispensed during patient visits. In some cases, dispensing information was not recorded. As it was not possible to accurately assess patient drug coverage between visits, we excluded any patient records that were missing dispensing information. In other instances, records of drugs dispensed were several times higher than expected; in many cases this also appeared related to data quality issues. Researchers manually examined the records of 108 patients with high rates of ARV over prescription to correct miscoded visit dates or exclude duplicated visit records.

3. Insufficient Uganda Cares MIS data were available to compare outcomes between UC-S and UC$\mathrm{MOH}$. Kitovu Mobile employs only task-shifted services, thus no internal comparison is possible.

4. TASO site-shifted services are intended to specifically address patient geographic preferences. Therefore exposure to task-shifting in TASO sites includes a large number of unmeasured variables. For instance, did patients who never attended CDDPs simply live closer to the central site? A study comparing various geographical locations of task-shifted studies would be useful in examining how site-shifting improves patient access.

Finally, it is difficult to draw definitive conclusions about the effects of task- and site- shifted services on client retention. Only stable TASO patients are eligible to acquire mobile services at the CDDP, which - by their very nature-are often more easily accessible to clients than brick-and-mortar facilities. Considering the preferential selection for stable clients, and self-selection by clients based on ease of access, it is not surprising that retention is better for clients who attend CDDPs. Thus, it difficult to tease apart those who remain in care longer because they can get services at CDDPs compared to those who have been shifted to the CDDP simply because they have remained in care.

In our multivariate analyses, we focused on a subset of stable, long-term ART clients in order to compensate for high rates of attrition during the early stages of care and to account for differences in treatment-seeking behaviors. The positive results demonstrated in this analysis suggest that site- and task-shifted services may indeed improve client retention and that further investigation of TASO's CDDP model is warranted.

\section{LIMITATIONS OF COSTING DATA}

Aside from the design limitations that prevent us from making any direct comparison of cost per visit between task-shifted and non-task-shifted models, the data were collected retrospectively and thus subjected to accounting errors and various data quality issues. These are common limitations of routine program data, especially data from local and grassroots organizations. However, we only used data from facilities that appeared to be comprehensive and we were able to impute some missing values by averaging values from the facilities with complete data.

For TASO and UC where the structure is more complicated, with staff from central level providing technical support to lower level facilities, the attribution of staff time to the various levels was based on gross estimates made by supervisors and not on timesheets. Further, health workers providing services to both ART and non-ART patients and their time spent on each type of patient, could be different, while we assumed that time spent on non-ART patients was the same as for ART patients. Also, although economic analysis would have been important to complement financial data, we were not able to collect information on the economic costs including time and money spent on waiting and transportation as well 
as other types of out-of-pocket payments.

Comparison of costs between the three ASOs should be done with caution for several reasons. The level of data completeness and data quality might be different across the three organizations. Nevertheless, these costing data are important for these three organizations and other AIDS organizations in Uganda as well as similar contexts for planning and budgeting. In addition, the study has demonstrated the feasibility of using existing data to estimate cost per ART patient visit while highlighting the need to strengthen local organization on collecting and using M\&E data. 


\section{DISCUSSION}

We studied three ASOs that differed in size and service delivery approaches. Kitovu Mobile is a small ASO providing ART in one region of Uganda using an outreach approach, thus its name-Kitovu Mobile. UC is a large ASO which is part of an international organization, AHF, that is providing ART in at least 11 African countries. In one UC model the clinics are entirely owned and managed by the ASO, while in the second model, UC provides drugs and supervision to existing MOH clinics that offer ART using $\mathrm{MOH}$ personnel and premises. TASO is another large ASO but it is indigenous to Uganda and provides ART using clinics that are entirely managed by the ASO. These three ASOs, with their varying approaches to task-shifting, provided us with a rich opportunity to explore a wide range of aspects of ART task-shifting. The study focused on the following key aspects that are vital for informing the formulation of guidelines and policies: what tasks are delegated, which cadre is involved in task-shifting, at what locations task-shifting is taking place, and what the implications of task-shifting are in terms of quality and outcomes of ART care.

This study shows that in the three ASOs almost all key tasks in ART, including determining eligibility, initiating ART, and dispensing ARVs, can be feasibly shifted to lower level cadres or lower level facilities. The study also shows a pattern of task-shifting where higher level HWs like physicians and clinical officers are mainly delegating out tasks; the lowest cadres—-the CHWs—are mainly being delegated tasks to; and the middle cadre, the nurses and counselor, are delegating out almost as much as they are being delegated to. Our data also show that task-shifting is taking place at both the centralized clinics and the decentralized clinics. These patterns of delegation have been documented in the literature (Callaghan, Ford, and Schneider 2010). However, our study helps highlight the fact that task-shifting is a continuum, implying that tasks are not being heaped onto one cadre, namely the nurses. While nurses are gaining the roles of determining eligibility and of initiating ART, they are also often delegating the traditional roles of triage and taking vital signs for patients.

In the 2008 WHO guidelines task-shifting (TS) is characterized in one dimension-the personnel involved in the task-shifting (WHO 2008). TS-I is where physicians delegate tasks to clinical officers and nurses; in TS-II clinical officers delegate tasks to nurses; in TS-III nurses delegate tasks to CHWs; and in TS-IV CHWs delegate tasks to expert patients. The data from our study adds two additional dimensions thus enriching the above definition-type of tasks delegated and type of venue to which ART services are delegated. These additional dimensions enable a further understanding of the nature and type of task-shifting. For example, we found that while in the UC-S model the pharmacy workers delegate ARV dispensing to the "HIV medic" (a CHW), in the TASO centralized model the pharmacy workers do not delegate ARV dispensing.

WHO recommends that countries should determine competencies for cadres to whom tasks are shifted and ensure that they receive requisite training to effectively perform these tasks (WHO 2008). In our study we observed gaps in the training of health workers. While the majority of cadres providing ART reported being trained in ART care, the proportion reporting training in specific competencies relevant to the tasks they were performing differed widely. Among professional health workers-doctors, 
clinical officers, and nurses - a low proportion of 30 percent to 50 percent reported training in the key competencies required of them: ART prescribing and dispensing. However, among the lay HWsHIV medics and expert clients - a higher proportion of 40 percent to 90 percent reported training in ART counseling and health education, which are the key competencies required of them. The findings are consistent with those of a survey of 44 health facilities across Uganda which found that among professional HWs who prescribed ARVs, 76 percent of doctors, 62 percent of clinical officers, and 62 percent of nurses were trained in initiating patients on ARVs (Lutalo et al. 2009).

Quality of ART care is one of the potential concerns about delegating tasks to lower level HWs. A number of studies have assessed the quality of ART care delivered by lower level health workers or CHW and have concluded that shifting ART tasks to these cadres did not negatively impact quality of care (Callaghan, Ford, and Schneider 2010). In our study we show that quality of care did not differ between HIV medics and other health workers (doctors, clinical officer, and nurses) working at the same type of clinic (UC). However, we observed a variation in quality by type of site-there was lower quality of care in the community-based settings (TASO-CDDP) compared with the facility-based clinics (TASO-C). However, it may not be accurate to make this comparison because the purpose of the client-provider interaction at the community site differs from that at the central health facility. At TASO-CDDP the purpose is to do a quick assessment and provide ARV refills for patients who are otherwise stable on ARVs. At TASO-C the purpose of the client-provider interaction is a thorough clinical review, either for clients who are in the initial phase of ART or those who have been referred from TASO-CDDP because of side effects or poor response to ARVs.

Quality of care can also be affected by work overload resulting in congestion, long waiting periods, and limited contact time with providers. Shifting some tasks to lower cadres is supposed to reduce the burden on higher level HWs thus enabling more patient contact time. For example Chung et al. 2008 reported that nurse-initiated ART reduces physician HIV-related workload by 78 percent. At the Reach Out Mbuya ART clinic in Uganda it was reported that after a task-shifting intervention, the overall median time spent at the clinic reduced from 206 to 83 minutes (Alamo et al. 2013). Our study has shown that the overall median time spent at the UC-S model was 48 minutes while the waiting time was 33 minutes, resulting in the patients spending 37.5 percent of the time at the clinic in contact with a provider. However, the other models in our study were less efficient in using the patient's time at the clinic in that the proportion of time spent in contact with a provider ranged from 6.7 percent to 15.6 percent. These findings suggest that some models of task-shifting can reduce waiting time and increase provider contact time for ART.

A key question is whether task-shifting affects patient outcomes. On-time attendance of scheduled drug-refill visits is associated with improved virologic treatment outcomes (El Khatib et al. 2011), thus eliminating treatment gaps presents a critical challenge for ART providers. However, in our study the data did not have reliable next scheduled medication pickup dates. Hence we used date-count projections for the next pick-up based on documented patient regimen and number of pills distributed at last visit. We selected 45 days' lateness as our cutoff point for loss to follow-up. This timeline falls within the definition as per a 2007 meta-analysis (Rosen, Fox, and Gill 2007) in which, "retention" referred to patients known to be alive and receiving highly active ART at the end of a follow up period. For non-retention, the studies in that review used cut-off points from 1 to 6 months late for a scheduled consultation or 
medication pick-up. In that meta-analysis 60 percent of patients were retained after 24 months. In our study 75.3 percent of the patients were retained in care at 24 months.

Only TASO provided data from ART services delivered to patients at a central facility, and through a community-based care model: the TASO-CDDPs. Bivariate analysis showed that retention at 12 and 18 months was significantly higher among those patients who attended any CDDP-based visits compared to those who did not. However, as discussed before given the pre-selection of CDDP patients and the ease of CDDP pick-up compared to visiting the central clinic, it is not surprising that the retention rate is better for those who attend CDDPs.

Consistent with previous costing analyses (Mdege et al. 2013; Menzies et al. 2011; McCoy et al. 2011; Bikilla et al. 2009; Martinson et al. 2009; Rosen, Long, and Sanne. 2008; Tagar et al. 2014), we found that ART drug-related expenses accounted for a significant portion of the total ART-related cost, followed by personnel and administrative costs. In particular, ART drugs accounted for nearly 50 percent of the total expenses for Kitovu Mobile and TASO models, while this proportion was nearly 70 percent for the UC model. Overall, the average annual cost per ART client among the three organizations $(\$ 331)$ was lower than previous similar analyses conducted among 5 PEPFAR countries (Botswana, Ethiopia, Nigeria, Uganda, and Vietnam; 2009 data), in which the median annual ART cost was $\$ 800$ (Menzies et al. 2011). The cost of ARV drugs has been reduced since then (Menzies et al. 2011; Harling and Wood 2007) which is likely the largest contributing factor for the lower annual ART cost in our study. In addition, these organizations have matured and become more efficient in serving increased numbers of ART clients (Harling and Wood 2007; Menzies, Berruti, and Blandford 2012). Lower ART costs suggest that future ART programs may become even less expensive, especially with the continuing reduction of drug costs.

The distribution of costs was quite comparable between Kitovu Mobile and TASO, which is consistent with previous study findings (Mdege et al. 2013; Menzies et al. 2011; Martinson et al. 2009; Tagar et al. 2014; Harling and Wood 2007; Larson et al. 2013). Kitovu Mobile employed a large number of expert clients (177) for their outreach activities, resulting in personnel costs comparable to that of TASO and higher than the UC's personnel costs. However, the interpretation of this finding should be cautious because UC employed a number of staff from the government and their salary data was not fully captured in this costing data. In addition, UC also used the facilities offered by the government free of charge, resulting in a much lower operational cost compared to Kitovu Mobile and TASO. The lack of personnel and operational costs within UC is likely the main reason contributing to the overall lower ART cost per client for UC model. This ultimately affects the cost distribution within the UC model. Further, even though ART expenses for UC accounted for nearly $70 \%$ of the total cost, the total UC ART cost in terms of absolute dollar value was quite comparable with Kitovu Mobile and TASO (data not shown).

In summary, the studies reviewed tend towards a general conclusion that task-shifting in any of the three dimensions - by type of provider, by level of facility, and by complexity of task delegated-mostly seems to result in the same level of outcomes when compared to the minimally task-shifted ideal of a doctor providing ART initiation and maintenance in a hospital. However, some studies suggest better results from task-shifted ART care while some others suggest poorer results from task-shifted ART care. Our study, which reviewed types and nature of task-shifting and examined the outcomes in three major ASOs in Uganda, has shown similar conclusions. When we compared task-shifting by level of facility we 
found that community-based settings were similar to facility-based clinics with regard to the following indicators: ARV knowledge of clients: provider performance in clinical assessment of patients, adherence counseling, and counseling about side effects. Our data also showed that community-based settings had better retention in ART than facility-based clinics. However, while overall client satisfaction was quite high in both types pf clinics, community-based settings performed less well than facility-based settings in providers addressing clients' complaints and in time efficiency. 


\section{CONCLUSIONS}

This study shows that in the three ASOs almost all key tasks in ART including determining eligibility, initiating ART, and dispensing ARVs can be feasibly shifted to lower level cadres or lower level facilities.

While the WHO has defined task-shifting using one dimension- the personnel involved in the taskshifting - the data from our study add two additional dimensions that could be used for a more descriptive definition - type of tasks delegated and type of venue to which ART services are delegated. Our study has also identified training gaps in specific areas of competence among professional and lay HWs currently providing ART in task-shifted services.

Our study has shown a number of variations in the process and outcomes of service delivery depending on how the task-shifted models were designed. Some of the models studied demonstrated efficient use of patient time while others did not. Our findings also suggest that the entirely outreach-based or more task-shifted model of Kitovu Mobile costs less per visit compared to the less task-shifted model of TASO. However, the distribution of costs between key cost elements such as personnel, drugs, lab tests, and operations differs between Kitovu Mobile and TASO and could partly explain the difference in cost per visit. The UC cost per visit is closer to Kitovu Mobile. It should, however, be noted that due to the challenges of compiling operational/office costs, the cost per visit at UC was likely an underestimate.

While our study has demonstrated that the quality of services was lower at the community-based clinics than at the health facility-based clinics our findings suggest that by employing a task-shifted, communitybased ART service model, TASO has expanded patient access to ARV refill resulting in high retention rates.

The findings from our study appear to agree with the available literature that task-shifting was associated with increased access and retention and reduced cost of ART care. Our data also showed that, in one ASO_-Uganda Cares, where we were able to compare provider performance-there was no difference in the performance of key ART tasks between LHWs to PHWs. Findings from this study provide important insight on costs and outcomes associated with task-shifting in facility-based versus outreach/communitybased service environments. 


\section{RECOMMENDATIONS}

- Consideration should be made to expand the description of task-shifted services along more than one dimension: the type of cadre, the type of facility and the type of task.

- When rolling out task-shifting in Uganda it will be important to conduct a training needs assessment of both the professional and LHWs and provide them with the required training in order to ensure competence and quality of care in task-shifted services. Given the likelihood of staff attrition, recruitment of new staff, and changes in guidelines, special attention should be paid to continued medical education for all cadres of staff involved in ART care.

- In our study we have shown that quality of care did not differ between HIV medics and other health workers (doctors, clinical officer, and nurses) working at the same type of clinic (UC). This suggests that the HIV medics are a cadre worth exploring for the scale-up of task-shifting.

- The reasons for the efficient use of patient time in models such as UC-S should be further explored and shared widely as promising practices that can strengthen other task-shifting models.

- Our data showed that community-based settings had better retention in ART than facility-based clinics. Given their low levels of drug coverage, yet high levels of retention, Kitovu Mobile should consider examining client viral loads to proactively identify any systemic concerns regarding viral suppression and drug resistance.

- Kitovu Mobile should examine current ART dispensing (or documentation) practices as, on average, the documentation shows that clients are receiving insufficient drugs to cover the interval between refills, and only 18.5 percent of clients achieved 95 percent drug coverage during their time in care.

- Future programs might benefit from examining the distribution of costs among the different elements of a task-shifted program in order to identify care packages that deliver the highest quality of care at the lowest cost.

- The collection of costing data to measure unit costs, cost-effectiveness, and cost-efficiency remains critical. ART service delivery sites in country would benefit from implementing a standardized cost data-capture tool or M\&E system that allows for comparison across sites. 


\section{REFERENCES}

Alamo, S. T. et al. 2013. "Strategies for optimizing clinic efficiency in a community-based antiretroviral treatment programme in Uganda," AIDS Behav. 17(1): doi:10.1007/s10461-012-0199-9.

Alwano, S. et al. 2012. "Task-shifting to community health workers: evaluation of the performance of a peer-led model in an antiretroviral program in Uganda," AIDS Patient Care STDS 26(2): 101-107. doi: 10.1089/apc.2011.0279.

Alibhai, A. et al. 2009. "Changes in quality of life for HIV patients in a community-based antiretroviral treatment program in rural Uganda." Paper presented at 5th IAS Conference on HIV Pathogenesis and Treatment, Cape Town, South Africa, 19-23 July. Abstract no. TUPED093.

Babigumira, J. B. et al. 2009. "Potential impact of task-shifting on costs of antiretroviral therapy and physician supply in Uganda," BMC Health Services Research 9: 192. doi:10.1186/1472-6963-9-192.

Bikilla, A. D., et al. 2009. "Cost estimates of HIV care and treatment with and without anti-retroviral therapy at Arba Minch Hospital in southern Ethiopia," Cost Eff Resour Alloc 7: 6.

Callaghan, M., Nathan Ford, and Helen Schneider. 2010. "A systematic review of task- shifting for HIV treatment and care in Africa," Human Resources for Health 8:8.

Castelnuovo, B., et al. 2009. "Improvement of the patient flow in a large urban clinic with high HIV seroprevalence in Kampala, Uganda,” International Journal of STD \& AIDS 20: 123-124.

Chung, J. et al. 2008. "Quantification of physician time saved in a task-shifting pilot program in Rwanda." Paper presented at XVII International AIDS Conference, Mexico City, Mexico, 3-8 August. Abstract no. WEAB0205.

Dambisya Y. M., et al 2012. "Policy and programmatic implications of task-shifting in Uganda: a case study," BMC Health Services Research 12: 61.

East, Central, and Southern African Health Community (ECSA-HC). 2010. "Task-shifting in Uganda: Case study.” Washington, DC: Futures Group, Health Policy Initiative, Task Order 1.

El-Khatib, Z., et al. 2011. "Adherence to drug-refill is a useful early warning indicator of virologic and immunologic failure among HIV patients on first-line ART in South Africa," PLoS One 6(3): e175.

Harling, G. and R. Wood. 2007. "The evolving cost of HIV in South Africa: changes in health care cost with duration on antiretroviral therapy for public sector patients," J Acquir Immune Defic Syndr. 45(3): 348-54.

Hiner, C. and G. M. Musokwa 2007. "Evaluation of AIDS Health Care Foundation's HIV medic programs in Uganda and Zambia," Final Report.

Kredo, T. et al. 2014. "Task-shifting from doctors to non-doctors for initiation and maintenance of antiretroviral therapy," Cochrane Database of Systematic Reviews 7: CD007331. DOI: 10.1002/14651858. CD007331.pub3. 
Kredo, T. et al. 2013. "Decentralising HIV treatment in lower- and middle-income countries," Cochrane Database of Systematic Reviews 6: CD009987. DOI: 10.1002/14651858.CD009987.pub2.

Larson, B.A., et al. 2013. "ART treatment costs and retention in care in Kenya: a cohort study in three rural outpatient clinics," J Int AIDS Soc.16: 18026.

Lutalo, I. M., et al. 2009. "Training needs assessment for clinicians at antiretroviral therapy clinics: evidence from a national survey in Uganda," Human Resources for Health 7: 76. doi:10.1186/1478-44917-76.

Martinson, N., et al., 2009. "Costs of providing care for HIV-infected adults in an urban HIV clinic in Soweto, South Africa," J Acquir Immune Defic Syndr 50(3): 327-30.

McCoy, K., et al. 2011. "Costs and outcomes of delivering antiretroviral therapy (ART) in the private and public sectors in Uganda: Final report." Boston: Health Initiatives for the Private Sector (HIPS) Project, Cardno Emerging Markets USA, Ltd.

Mdege, N.D., et al. 2013. "The effectiveness and cost implications of task-shifting in the delivery of antiretroviral therapy to HIV-infected patients: a systematic review," Health Policy Plan 28(3): 223-236.

Menzies, N.A., et al. 2011. "The cost of providing comprehensive HIV treatment in PEPFAR-supported programs," AIDS 25(14): 1753-1760.

Menzies, N. A., A. A. Berruti, and J. M. Blandford. 2012. "The determinants of HIV treatment costs in resource limited settings," PLoS One 7(11): e48726.

Ministry of Health (MOH). 2011. Uganda AIDS Indicator Survey 2011. Kampala: Ministry of Health.

Ministry of Health (MOH) [Uganda] and ORC Macro. 2006. Uganda HIVIAIDS Sero-behavioural Survey 2004-2005. Calverton, Maryland, USA: Ministry of Health and ORC Macro.

Rosen, S., M. P. Fox, and C. J. Gill. 2007. "Patient retention in antiretroviral therapy programs in subSaharan Africa: a systematic review," PLoS Medicine 4(10): e298.

Rosen, S., L. Long, and I. Sanne. 2008. "The outcomes and outpatient costs of different models of antiretroviral treatment delivery in South Africa," Trop Med Int Health. 13(8): 1005-1015.

Stilwell, B. 2007. "Guideline for incorporating new cadres of health workers to increase accessibility and adherence to antiretroviral therapy." Chapel Hill, NC: The Capacity Project, IntraHealth International, Inc.

Tagar, E., et al. 2014. "Multi-country analysis of treatment costs for HIV/AIDS (MATCH): facilitylevel ART unit cost analysis in Ethiopia, Malawi, Rwanda, South Africa and Zambia," PLoS One 9(11): e108304.

Uganda Cares. 2003. "First year progress report, July 2003.” Masaka, Uganda: Uganda Cares. Uganda AIDS Commission. 2011. UNGASS Country Progress Report Uganda. Kampala: Uganda AIDS Commission. Last accessed 12 June 2012 at https://www.unaids.org/en/dataanalysis/ monitoringcountryprogress/progressreports/2010countries/uganda_2010_country_progress_report_ en.pdf. 
Uganda AIDS Commission. 2014. Uganda HIV and AIDS Country Progress Report, 2013. Kampala: Uganda AIDS Commission. www.unaids.org/sites/default/files/en/dataanalysis/knowyourresponse/countr yprogressreports/2014countries/UGA_narrative_report_2014.pdf

Vasan, et al. 2009. "Agreement between physicians and non-physician clinicians in starting antiretroviral therapy in rural Uganda," Human Resources for Health 7: 75. doi:10.1186/1478-4491-7-75.

Wanyenze, R. K., et al. 2010. "Evaluation of the efficiency of patient flow at three Ugandan HIV clinics," AIDS Patient Care STDs 24: 441-446.

World Health Organization (WHO) 2007. "Task-shifting to tackle health worker shortages." Geneva: WHO. Accessed on 12 June 2012 at http://www.who.int/healthsystems/task_shifting_booklet.pdf.

WHO. 2008. "Task-shifting: rational redistribution of tasks among health workforce teams: global recommendations and guidelines." Geneva: WHO.

WHO 2012. "Technical update on treatment optimization: use of efavirenz during pregnancy: a public health perspective." Geneva: WHO.

Zachariaha, R. et al. 2009. "Task-shifting in HIV/AIDS: opportunities, challenges and proposed actions for sub-Saharan Africa," Transactions of the Royal Society of Tropical Medicine and Hygiene 103: 549-558. 
U.S. Agency for International Development I 300 Pennsylvania Avenue, NW

Washington, DC 20523

Tel: (202) 7I2-0000

Fax: (202) 216-3524

www.usaid.gov 